\title{
Ultra-high speed video observations of intracloud lightning flash initiation
}

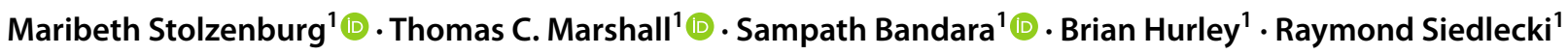

Received: 2 February 2021 / Accepted: 11 May 2021 / Published online: 25 May 2021

(c) The Author(s) 2021

\begin{abstract}
This study describes results from video observations of five intracloud flashes located $\leq 20 \mathrm{~km}$ from the camera and recorded with $6.1 \mu$ s exposure time and $6.66 \mu$ s frame intervals. Video data are supported with electric field change (E-change) and VHF measurements, with emphasis on the flash initiating event (IE) and initial breakdown (IB) stage. In four of the five flashes, the IE is accompanied by weak luminosity, $\leq 5 \%$ above background, lasting for 300-500 $\mu$ s. Two of these four IEs were positive Narrow Bipolar Events (NBEs) with VHF powers of 43 and $990 \mathrm{~W}$; these are the first (known) data showing visible light detected with a positive NBE. Two other IEs with weak luminosity had powers of 0.5 and $1 \mathrm{~W}$, and the IE with no detected luminosity had a VHF power of $3 \mathrm{~W}$. A typical IB cluster consists of several narrow pulses and one classic pulse in E-change data (along with many VHF pulses), and each example flash has 2-10 IB clusters in the first 5-50 ms. The luminosity of IB clusters was substantially greater than IE luminosity, ranging from 10 to $40 \%$ above background in four examples, while for one flash with 10 IB clusters, the luminosity range was 35-360\% above background (average 190\%). Luminosity durations of IB clusters were $520-1750 \mu$ s with average $1210 \mu$ s. For both IEs and IB clusters, increases in the detected luminosity were closely timed with substantial VHF emissions and decreased when VHF emissions weakened.
\end{abstract}

\section{Introduction}

The primary emphasis of this study is the visible light observed during the initiating event (IE) and initial breakdown (IB) stage of typical intracloud (IC) lightning flashes. As known from studies through the past several decades, the IB stage of a flash includes bipolar and unipolar pulses, typically seen in electric field change (E-change) data, that are called IB pulses or IBPs herein. IB pulses can be classed into two types: classic IBPs have longer durations (10-50 $\mu$ s or longer) and larger amplitudes than narrow IBPs (e.g., Nag et al. 2009; Marshall et al. 2019; Shi et al. 2019). For the purposes of this paper, we use the term IC flash to mean a typical IC flash, that is, one which initiates in negative electric field and in which the negative (end of the) initial leader propagates mainly upward.

\section{Responsible Editor: Rubin Jiang.}

Maribeth Stolzenburg

mstolzen@phy.olemiss.edu

1 Department of Physics and Astronomy, University of Mississippi, University, MS, USA
It is of intrinsic interest to understand how IC flashes begin, as well as to compare them to the initiation of cloud-to-ground (CG) flashes. There are fundamental reasons why the initiation process might occur differently in the two flash types, including that IC flashes typically initiate at higher altitudes and in a vertical electric field (E) that has negative polarity, opposite that of CG flash initiations (e.g., Coleman et al. 2003). Perhaps the most significant difference between the early stages of lightning flash development was noted by Kitagawa and Brook (1960), who found that the interval between successive classic IB pulses had a mean value of $680 \mu$ s for IC flashes versus a mean of only $80 \mu$ s for CG flashes. Weidman and Krider (1979) found a similar disparity by flash type, with mean intervals of $780 \mu$ s (IC) versus $130 \mu$ s (CG). More recent IC flash studies have shown that the typically long intervals between classic IB pulses result in the initial IC development occurring in "bursts" with each burst having several narrow IB pulses and one or two classic IB pulses (e.g., Marshall et al. 2013) and producing significant observable light (e.g., Stolzenburg et al. 2013). Marshall et al. (2013) describe the E-change signature at the beginning of typical IC flashes as "The IBPs primarily occur in short $(<750 \mu \mathrm{s})$ bursts of two to five pulses... with 
successive bursts separated by 1-5 ms." Herein we adopt the terminology of Shi et al. (2019) and call a single burst containing one or more narrow IB pulses and one or more classic IB pulses an "IB cluster." In typical IC flashes, successive IB clusters occur at successively higher altitudes (Marshall et al. 2013, 2019; Shi et al. 2019). Recent work by Scholten et al. (2021) shows imaging data for two inverted-polarity IC flashes. (Unlike typical IC flashes, inverted polarity IC flashes are those in which the initiation and IB stage are like that of a normal negative CG flash in that they initiate in a positive electric field and the negative leader initially propagates downward.)

The IE of most IC flashes is a low power $(<1 \mathrm{~W})$, shortduration $(\leq 1 \mu \mathrm{s})$ VHF pulse that often does not have a coincident E-change pulse (e.g., Marshall et al. 2014, 2019; Lyu et al. 2019). Alternatively, the IE of some IC flashes is a positive Narrow Bipolar Event or NBE (e.g., Rison et al. 1999, 2016; Wu et al. 2014). An NBE is a particular class of discharge first described by Le Vine (1980) and Willett et al. (1989) as an isolated in-cloud process that produces a large-amplitude, bipolar E-change pulse with a typical duration of $20-40 \mu$ s and a coincident, powerful pulse in the HF and VHF radio bands. (Note that NBEs are also referred to as compact intracloud discharges (CIDs) in some literature, e.g., Nag et al. (2010) and Leal et al. (2019).)

Although IC flashes are common, optical data with timecorrelated E-change observations for their initiation are relatively rare. Most prior studies using high-speed video data have focused on the IB stage of CG flashes (e.g., Stolzenburg et al. 2013, 2014; Campos and Saba 2013). High-speed video data of four hybrid IC-negative CG flashes with ICtype IB clusters were described in Stolzenburg et al. (2016), which focused on eight very large-amplitude IB pulses. For those IB pulses the video intensity "increased sharply for 80-220 $\mu$ s" after the E-change peak with total visible durations of 300-800 $\mu$ s. Using an avalanche photodiode with a response time of $10 \mathrm{~ns}$, Wilkes et al. (2016) examined the full-sky luminosity of classic IB pulses during 30 CG and 37 IC flashes. This fast response time revealed small but distinct differences between CG-type and IC-type classic IB pulses, including a much longer delay between the beginning of E-change IB pulse and the onset of the corresponding luminosity (mean values of $8 \mu$ s versus $34 \mu$ s for CG vs IC) and slower luminosity rise times for classic IB pulses (mean values of $25 \mu$ s versus $59 \mu$ s for CG vs IC) (Wilkes et al. 2016). In what seems to be the only study of luminosity mentioning the flash IE, Stolzenburg et al. (2020) detected "a weak bipolar pulse $60 \mu$ s prior to the first initial breakdown (IB) pulse" which was accompanied by evidence of weak luminosity in one CG flash. Other studies of luminosity during the IB stage of CG and IC flashes apparently either did not recognize or did not detect the IE, and none comment on observing luminosity with the IE. Clearly, more optical data are needed to study IC flash initiation and to better describe the differences seen between IC and CG initiations.

For this study, we obtained ultrahigh-speed video, E-change, and VHF data during the IE and IB stage in IC flashes. To the best of our knowledge, no prior study has investigated the luminosity of IEs in IC flashes. The camera applied in this work has better light sensitivity than the highspeed video cameras previously used for our lightning studies, which is important since IC flash initiations are likely deep inside the cloud relative to the ground-based camera. This paper describes results for the first several milliseconds of five IC flashes. One main finding is that the IE of the five IC flashes did not have strong luminosity: no visible luminosity was detected for one IE and very weak visible luminosity was coincident with four IEs. Two IEs were positive NBEs, and three IEs were weak events. Another main finding is that the duration of the strong visible luminosity associated with each IB cluster is closely associated with the VHF radiation emitted during the IB cluster; the luminosity and VHF radiation usually begin (on average) $\sim 300 \mu$ s before the classic IB pulse peak and continues (on average) for $\sim 1000 \mu$ s after the classic IB pulse peak.

\section{Data sources}

The array of electromagnetic instruments used for this study was similar to that described in Marshall et al. (2019). Electric field change (E-change) data were obtained with flatplate fast antenna (FA) sensors of the sort used by Kitagawa and Brook (1960), Weidman and Krider (1979), and others since. Our FA sensors had decay time constant of $10 \mathrm{~ms}$ and bandwidth of $16 \mathrm{~Hz}-2.6 \mathrm{MHz}$. The VHF data were acquired using a LogRF sensor measuring power in the $186-192 \mathrm{MHz}$ band on a logarithmic scale. The time derivative of the electric field was measured with a dE/dt sensor having bandwidth $0-2.5 \mathrm{MHz}$; integration over short time periods of the dE/dt data (e.g., Jerauld et al. 2008) can yield a better depiction of small E-change pulses than seen in the FA data. Electromagnetic data from the three sensors were obtained at seven stations within $45 \mathrm{~km}$ of Oxford, MS, USA. (Figures including station locations are shown later.) Approximate terrain altitude in the region is $110-160 \mathrm{~m}$ MSL. Data were recorded in triggered mode for $400 \mathrm{~ms}$, digitized at $10 \mathrm{MegaSamples/s,} \mathrm{whenever} \mathrm{a} \mathrm{pulse} \mathrm{with} \mathrm{amplitude} \mathrm{above}$ a threshold was detected by the FA at the site. Locations for individual events were determined using the time-of-arrival 'Position By Fast Antenna' technique (Karunarathne et al. 2013), and also using the same technique with multi-station integrated dE/dt data, as described in Bandara et al. (2019). The latter locations are identified in figure legends as $\int d E / d t$. For some of the IC flashes presented herein, there were insufficient triggered data to derive PBFA locations because there 
was not a large-amplitude E-change pulse detected at some stations during the flash. Available LIghtning NETwork (LINET) data were used for these flashes when detected; LINET (e.g., Betz et al. 2004, 2008) is a system of magnetic field change sensors at VLF/LF (5-200 kHz) that locates and discriminates between in-cloud and ground events. Using the methods described in Bandara et al. (2019), the VHF powers of individual events were determined using the Friis equation, and the E-change amplitude of individual events was range normalized to $100 \mathrm{~km}$ (and labeled as $E_{100 \mathrm{~km}}$ ). Figures showing time series data are labeled with the flash time in (decimal) seconds past $00 \mathrm{UT}$, except in two plots where the time is in ms past a beginning time.

In addition to the above instrumentation, three video cameras were operated during the experiment from seventh floor windows of a building on the University of Mississippi campus. The data presented herein were acquired with a Vision Research Phantom ${ }^{\mathrm{TM}}$ v2512 ultrahigh-speed video camera. According to the camera manufacturer's datasheet (see www.phantomhighspeed.com), this camera has light sensitivity (ISO) of 32,000 Daylight and 100,000 Tungsten. Its spectral response is $>0.02 \mathrm{~A} / \mathrm{W}$ across wavelengths from 350 to $1060 \mathrm{~nm}$, with peak response at $670 \mathrm{~nm}$. Importantly, this camera has average response of $\sim 0.06 \mathrm{~A} / \mathrm{W}$ between 350 and $400 \mathrm{~nm}$. (Spectral response is the ratio of photocurrent output to radiant energy input on the sensor at the range of wavelengths. The lower limit of the manufacturer's available spectral response measurement is $330 \mathrm{~nm}$.) The high light sensitivity of this camera is critically important to the study of IC flash initiation, since these initiations are typically higher and deeper inside the cloud than the CG flash initiations studied previously (e.g., Stolzenburg et al. 2013, 2014,2020 ). For the data presented herein, the camera was operated at 150,126 frames/s, or $6.66 \mu \mathrm{s}$ frame interval, and image exposure of $6.1 \mu \mathrm{s}$. Frame resolution used was $256 \times 352$ pixels, and the camera was fitted with a $14-\mathrm{mm}$ focal length lens. Because the intensity enhancements are diffuse within the images in the examples, they are difficult to see in reproduction. Hence, we present cumulative intensity, determined by summing all the pixel intensity values (14-bit, saturated intensity is 16384) in each image then dividing by the total number of pixels $(90,112)$. Since the field of view of the camera is large (as given below, about $8-9 \mathrm{~km}$ across by $10-12 \mathrm{~km}$ above ground at the ranges to these flashes), the image frame includes light scattered from the obscured sources by cloud and precipitation particles within the frame. Wilkes et al. (2016) discuss the effect of optical scattering on light signal received from diffuse sources, and Stolzenburg et al. (2016) have estimated the delay in light arrival caused by scattering in similar video data (where the scattering angle is $<10^{\circ}$ ) may be up to $3 \mu$ s. The effect of scattered light for distinct point sources and linear features can be a slight delay in timing between when the brightest pixels reach maximum and when the cumulative intensity in the frame reaches its maximum; from a close comparison of observed distinct light sources (e.g., bright channels), we estimate this timing delay of the cumulative intensity is less than $20 \mu \mathrm{s}$, or about three frames, for later parts of the flashes (a) base scan PPI, 2019-06-20 0452:58 UT

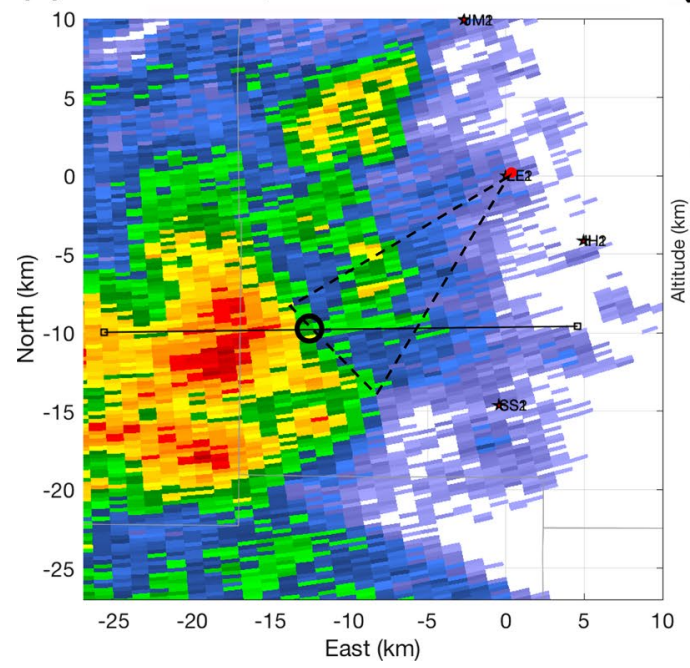

(b) west-to-east cross-section (at $\mathrm{y}=-10 \mathrm{~km}$ )

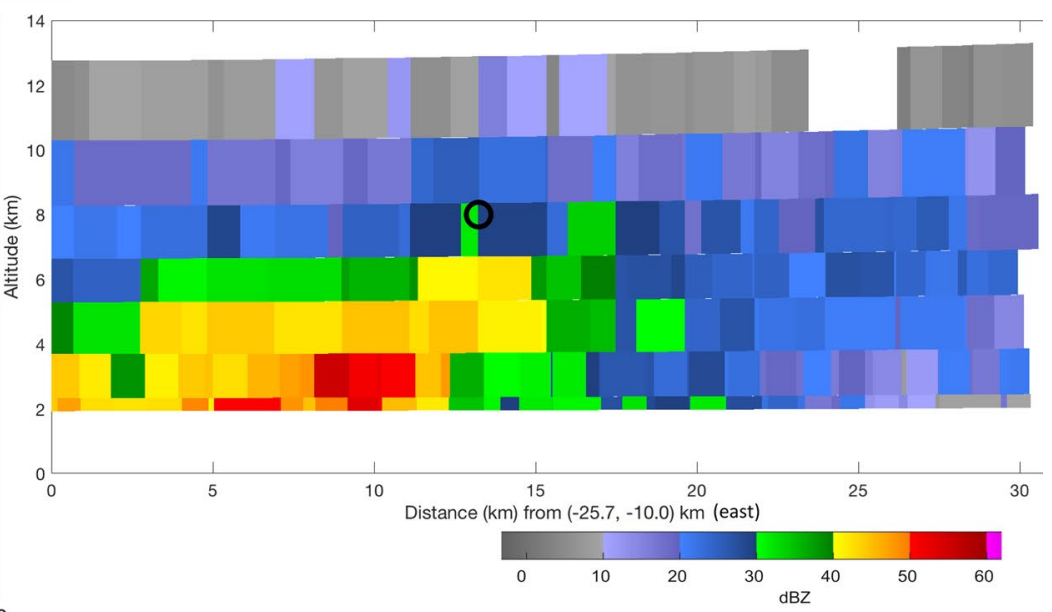

Fig. 1 Radar reflectivity for Example 1, IC flash at 0450:20 UT on 20 June 2019. Grid origin is located at the 'EE' sensor site in Oxford, MS. Radar data are from KNQA in Memphis, TN, approximately $115 \mathrm{~km}$ distant. a Plan view of low-level scan, with locations of camera site (red circle), camera field-of-view (dashed), and approximate flash beginning (black circle). Labeled black stars are electromagnetic sensor sites, and blackline indicates cross-section location. b Radar cross-section through storm, near location of IC flash beginning, with approximate initiation location indicated by black circle 


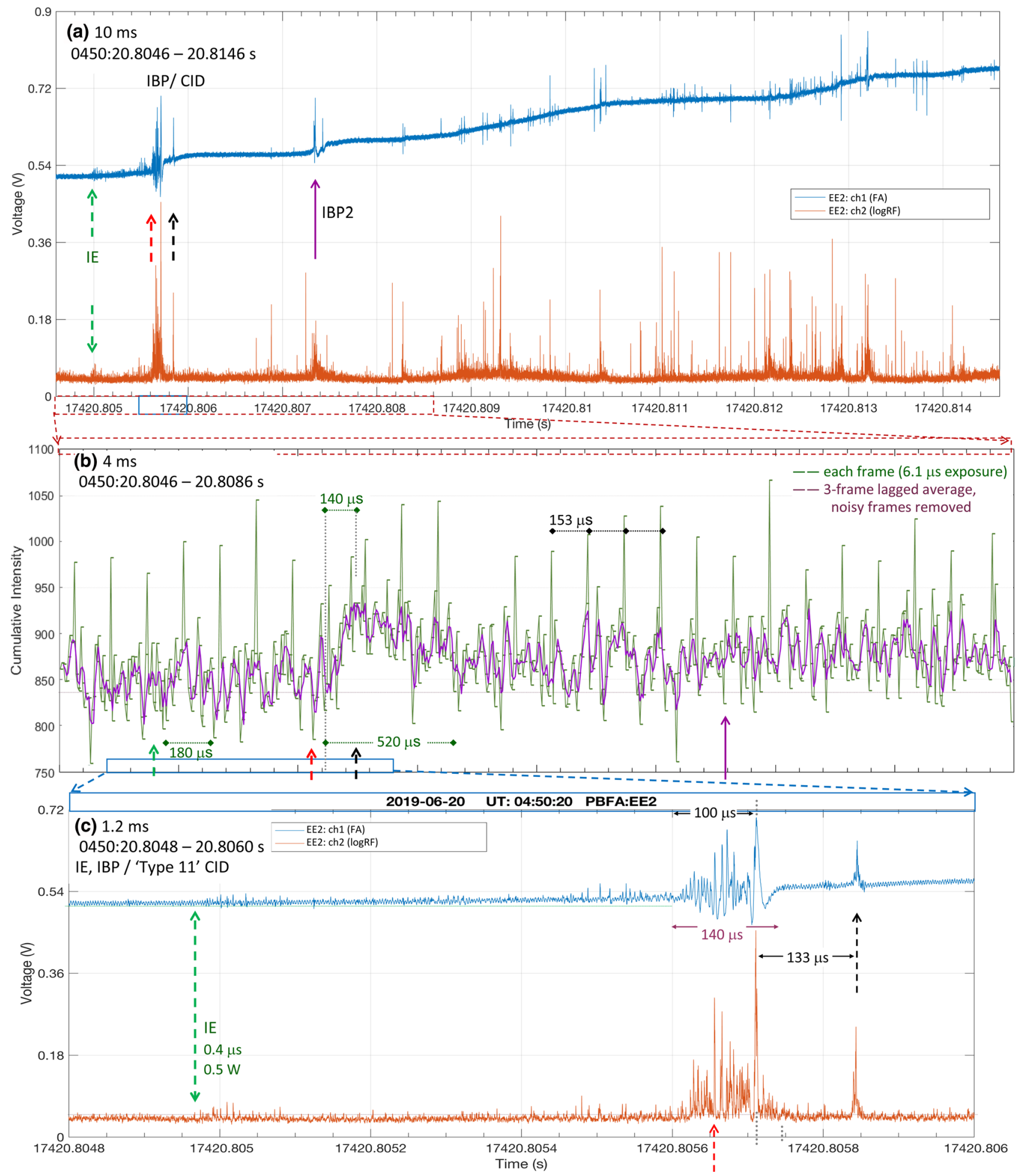

studied herein. For diffuse events, like all the events discussed in this study, scattered light is part of the luminosity signal and hence may slightly extend the duration of weakly enhanced luminosity (by $20 \mu$ s or less) but does not otherwise influence the relationship between luminosity and the electromagnetic data. Importantly, the onset of the luminosity increase is not delayed significantly by scattering.

For the purposes of analysis, intensity enhancements are defined herein as periods when the cumulative intensity reaches at least 3\% above background, averaged through three frames $(20 \mu \mathrm{s})$, over a period of at least 
4Fig. 2 Beginning of IC flash Example 1 at 0450:20 UT on 20 June 2019, approximately $16.0 \mathrm{~km}$ distance (17.9 km slant range) from camera. a First $10 \mathrm{~ms}$ of E-change (FA, ch1) and VHF (logRF, ch2) data. Green arrow marks the IE of the flash, red and black arrows indicate beginning of large oscillations and last (trailing) IBP of the first IB cluster, and purple arrow marks the classic IBP of the second IB cluster. Red and blue rectangles along the time axis indicate periods covered by lower figure parts. b Cumulative intensity from video data for the first $4 \mathrm{~ms}$, including the same four arrows from Fig. 3a). Although noisy, slight increases in intensity (peaks are 5\% and $11 \%$ above average background, indicated by horizontal line) are detected indicating light associated with the IE and first IB cluster; durations above background are $180 \mu \mathrm{s}$ and $520 \mu \mathrm{s}$, and time of increase to light peak with IBP is $140 \mu$ s. (Noise spikes of unknown origin occur every 23rd frame, $153 \mu$ s interval.) Noisy frames are removed from the 3-frame lagged average values, purple curve. c FA and $\operatorname{logRF}$ data for $1200 \mu \mathrm{s}$ of the IE and first IB cluster. VHF pulse duration and power are given for the IE and dashed green horizontal indicates departure of E-change data from background (IEC). In the IB cluster, FA data resemble the 'Type 11' CID waveform with $\sim 140 \mu$ s duration of E-change oscillations. The E-change and VHF peaks are coincident, near the start of rising light intensity; these peaks follow the onset of oscillations by about $100 \mu$ s and precede the trailing pulse by $133 \mu$ s. The light intensity peak is nearly coincident with the trailing

12 frames $(80 \mu \mathrm{s})$. Enhancement durations are considered to start when the cumulative intensity departs from the background and end (approximately) when values return to near background. Because there is recurring 'speckle' noise in some of the cumulative intensity data, we have determined a 3-frame 'lagged average' (average of frame and the two previous frames, for $20 \mu$ s effective exposure time) in which the noisy frames have been removed before averaging (i.e., averages are over two frames around noise spikes). These lagged average curves are included for periods with weak enhancements.

Data collection occurred from late May to early July, 2019. The data presented herein were acquired on two nights, June 20 and 22 (UT), when short convective lines were near the camera site. Reflectivity data from the closest NWS radar (KNQA, Memphis, TN, approximately $115 \mathrm{~km}$ from the camera site) are used to describe the general storm location and precipitation structure.

\section{Observations}

We present five examples for which ultrahigh-speed video data exist during the beginning of IC flashes. These are some of our best cases in terms of completeness and quality of the video data showing luminosity detected with IEs and IB clusters. We investigate IE luminosity in all five examples. In Examples 1-4, we also focus on the possible positive NBEs that occur first or very early in these flashes. In Examples 3-5, we examine the light signatures detected with IB clusters. Additional cases in the dataset are undergoing analysis, and a more complete statistical study of luminosity during IC flash initiation is planned for a later paper.

\subsection{Example 1}

The first IC flash we consider occurred at 0450:20 UT on 20 June 2019. Figure 1 shows radar reflectivity data for the time. This flash was approximately $16 \mathrm{~km}$ horizontal distance southwest of the camera; the imaged area at this distance is $8.2 \mathrm{~km}$ across by $10.3 \mathrm{~km}$ above ground. Using the estimated slant range of $17.9 \mathrm{~km}$ to the approximate initiation location (at $8 \mathrm{~km}$ altitude) yields imaged area of $9.2 \mathrm{~km}$ across and $11.6 \mathrm{~km}$ above ground. Locations of pulses for Example 1 could not be determined due to insufficient triggered sensors, and LINET did not detect any events with this flash. We estimate the general area of visible activity based on the storm location, camera angle, and surrounding flashes located with PBFA or LINET. The overall duration of this flash in E-change data was about $480 \mathrm{~ms}$.

Electromagnetic and video light intensity data from the beginning of this IC flash are displayed in Fig. 2. Figure $2 \mathrm{a}$ shows the first $10 \mathrm{~ms}$ of E-change (FA, ch1) and VHF ( $\log R F, c h 2)$ data for Example 1. The initiating event (IE) of the flash occurred at 0450:20.804975 UT or 17,420.804975 s from midnight (marked by vertical, dashed, green arrow in Fig. 2). The weak IE was a VHF pulse with a duration of $0.4 \mu$ s and an estimated power of $0.5 \mathrm{~W}$ (Fig. 2c). (Although the pulse identified as the IE is small, it was detected at two separate sites, $43 \mathrm{~km}$ apart, hence we would not interpret it as local noise. Also, by definition, we have interpreted the IE as the first detected pulse in Example 1; we cannot be sure there was not an earlier, undetected VHF signal that may have been the real IE of the flash.) There was no coincident E-change pulse with the VHF pulse of the IE, but starting at the time of the IE, the E-change data begin monotonically increasing (Fig. 2c); this increase is known as an Initial E-Change or IEC (e.g., Marshall et al. 2014; Chapman et al. 2017). The IEC is caused by a weak current flow, and the beginning of the IEC is an independent indication of the time of the IE. The cumulative light intensity time series (Fig. 2b) shows that the video data begin quite dark and noisy, with values averaging about 830 units. (The noise in the cumulative intensity data is noticeable only in very low light situations and is mainly due to the sharp upward spikes that occur every 23 rd video frame or every $153 \mu \mathrm{s}$, as marked in Fig. 2b.) Starting about $60 \mu$ s after the IE (green arrow), the cumulative intensity (Fig. 2b) increases to about $5 \%$ above background in $\sim 100 \mu \mathrm{s}$, then decreases for total duration of about $180 \mu \mathrm{s}$. However, given the fluctuations in this low light observation, it is not certain that the IE caused the $5 \%$ increase. In other words, this IE is coincident 

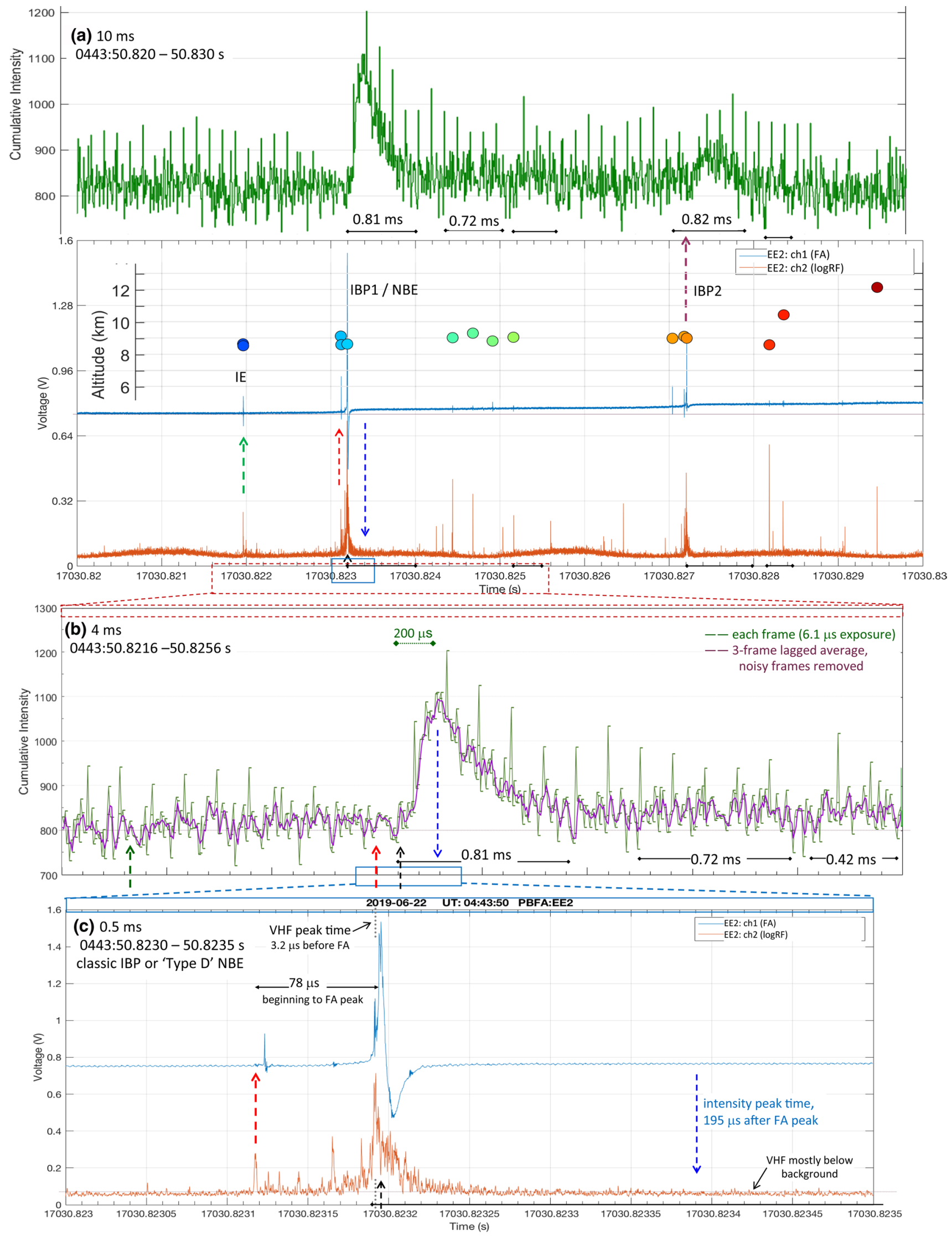
4Fig. 3 Beginning of IC flash Example 2 at 0443:50 UT on 22 June 2019, at $15.0 \mathrm{~km}$ distance (17.2 km slant range) from camera. a First $10 \mathrm{~ms}$ of E-change (FA, blue curve) and VHF (logRF, red curve) data; colored circles, inset, are located pulse altitudes (see Fig. 4). Green curve (above) is cumulative intensity data. Green arrow indicates the initiating event (IE). The first IB cluster (starting at red arrow) has two located narrow IBPs, and a classic IBP or an NBE (black arrow is FA peak time); the intensity peak during the first IB cluster is marked with blue arrow. Purple arrow marks the classic IBP of the next IB cluster. Black horizontal bars indicate times of intensity above average background. b Cumulative intensity (per frame and 3-frame lagged average, cleaned) for first $4 \mathrm{~ms}$, with arrows from a. A distinct increase in intensity (peak $37 \%$ above background) indicates light emission began with the IBP/NBE; intensity rises above background for $0.81 \mathrm{~ms}$, beginning just before the FA peak. Slightly increased intensities (3-5\%) are visible after the IE only as increased average background. c $500 \mu$ s of E-change and VHF data for the first IB cluster, with arrows as in a. The main bipolar pulse is a classic IBP or 'Type D' positive NBE, with VHF peak (black dotted vertical) slightly preceding (by $3.2 \mu \mathrm{s}$ ) the E-change peak and both peaks occurring near the start of the rise in intensity. Event location was $14.4 \mathrm{~km}$ distance from this sensor and at $8.9 \mathrm{~km}$ altitude

with either no visible luminosity or only a weak luminosity increase of $\leq 5 \%$ above background.

In the first $130 \mu$ s after the weak IE there are many small VHF pulses (powers $<1 \mathrm{~W}$, durations $<2 \mu \mathrm{s}$ ); some of the VHF pulses have coincident E-change pulses (narrow IB pulses) with $E_{100 \mathrm{~km}}$ amplitudes $<0.1 \mathrm{~V} / \mathrm{m}$; this development is similar to that of an IC flash initiation studied by Marshall et al. (2019, see their Fig. 4b). Small pulses continue intermittently for an additional $\sim 548 \mu$ s (to $17,420.805653 \mathrm{~s}$, marked with vertical red arrow in Fig. 2), after which oscillations detected by both sensors become abruptly larger for $60 \mu \mathrm{s}$. The series of much larger oscillations leads up to the largest pulse in both E-change and VHF data at 17,420.805711 s. This bipolar E-change pulse may be the first classic IB pulse of the flash; it has an estimated VHF power of $12 \mathrm{~W}$ and an $E_{100 \mathrm{~km}}$ amplitude of $0.84 \mathrm{~V} / \mathrm{m}$. About $133 \mu$ s after the largest E-change pulse, at the end of a series of small VHF pulses and quiet E-change data, there is a trailing pulse of $20 \mu$ s duration detected in both sensors at 17,420.805845 (black arrow in Fig. 2). The first IB cluster of this flash is mainly comprised of the IB pulses between the red and black arrows in Fig. 2; Fig. 2a shows that there is an electrostatic E-change (an upward step) during the time between the red and black arrows that must be associated with significant charge motion.

The E-change waveform of the largest IB pulse, expanded in Fig. 2c, also fits the character described by Leal et al. (2019) as a 'Type 11' CID, having $\sim 100 \mu$ s of preceding oscillations and total duration of $\sim 140 \mu$ s (marked in Fig. 2c). The last, bipolar part of this event also fits characteristics of a positive NBE, particularly in the fact that the relatively strong VHF emission peak is coincident with the large E-change peak, as documented for positive NBEs by Bandara et al. (2020). Thus this bipolar pulse may be a positive NBE rather than a classic IB pulse. The bipolar pulse's estimated VHF power of $12 \mathrm{~W}(10.9 \mathrm{dBW})$ is in good agreement with the powers of NBEs initiating ten IC flashes found by Rison et al. (2016, their Fig. 5), which included six positive NBEs with powers of 1-15 W. However, as seen in subsequent examples herein, a classic IB pulse can also have a power of $12 \mathrm{~W}$, so without further information, we cannot decide if this largest bipolar E-change pulse is a classic IBP or a positive NBE. We note that Rison et al. (2016) showed one example similar to our Example 1: an IC flash began with a positive NBE (their NBE2) and was followed by another positive NBE in the first IB cluster, though in their case the second NBE was not the largest bipolar pulse in the IB cluster nor was it preceded by the large-amplitude E-change oscillations characteristic of a Type $11 \mathrm{CID}$. Overall, the beginning of this IC flash is more complicated than any previously reported.

After the IE, no clear indication of luminosity can be gleaned from the cumulative intensity data during the period of initial small oscillations in the E-change and VHF data (between green and red arrows in Fig. 2a, b). However, there is a clear increase in intensity detected with the largest VHF pulses. The intensity rise becomes obvious within $70 \mu \mathrm{s}$ after the red arrow; the luminosity increase is essentially coincident with the series of largest E-change and VHF peaks. The cumulative intensity peak ( 930, 11\% above background) occurs about $140 \mu \mathrm{s}$ ( 21 frames) later, at approximately the time of the trailing E-change pulse and the ending spike of substantial VHF emission. The decay in the cumulative intensity is much slower than its rise, occurring while the VHF activity also slowly returns to the background level. The duration of the luminosity enhancement associated with the first IB cluster is about $520 \mu \mathrm{s}$ (marked in Fig. 2b).

In Example 1, the classic IB pulse of the second IB cluster occurred $1.63 \mathrm{~ms}$ after the largest E-change pulse of the first IB cluster. The time of this IB pulse is indicated (purple arrow) in Fig. 2. A weak increase in video-detected intensity associated with the IB pulse is visible in Fig. 2b. Based on the much stronger light observed with IB pulses in other IC flashes (shown below), it seems likely that this IB pulse was either above the video frame or mostly obscured from the camera view by intervening clouds.

Later during Example 1 flash, there was weak luminosity detected with four large IBPs in the first $13.5 \mathrm{~ms}$. This brief IB stage was followed by a quiet period of about $178.1 \mathrm{~ms}$ duration, after which the flash re-started and there were then 12 intermittent periods of diffuse light detected through $50 \mathrm{~ms}$, each associated with a brief series of IB pulses. Within the camera field of view, beginning $62.7 \mathrm{~ms}$ after the second start of the flash (and $254.3 \mathrm{~ms}$ after the IE), IC leader propagation and IC strokes were visible at intervals in the video data over a period lasting more than $203.5 \mathrm{~ms}$. 


\subsection{Example 2}

The second example of luminosity data during the beginning of an IC flash is shown in Fig. 3. This flash occurred at 0443:50 UT on 22 June 2019 and lasted more than $720 \mathrm{~ms}$. It began $15.0 \mathrm{~km}$ southeast of the camera site, near the northern edge of a small multicell storm which rapidly developed out of an outflow boundary (from an earlier mesoscale storm complex much farther north). The imaged area of the camera at this distance is $7.7 \mathrm{~km}$ across by $10.3 \mathrm{~km}$ above ground; at the slant range $(17.2 \mathrm{~km})$ to the initiating event, the imaged area is $8.8 \mathrm{~km} \times 11.8 \mathrm{~km}$. Early pulse locations overlaid on radar reflectivity data in Fig. 4 indicate flash initiation was at mid-level in $35 \mathrm{dBZ}$ echo and ascended into about $15 \mathrm{dBZ}$. The camera view to the flash initiation was through about $4 \mathrm{~km}$ of weak echo depth. Radar echo top of the storm was about $14 \mathrm{~km}$ altitude. In the electromagnetic data the first detected event (the IE) was located at $8.6 \mathrm{~km}$ altitude. Based on its range-normalized E-change amplitude $(0.3 \mathrm{~V} / \mathrm{m})$, E-change duration $(4 \mu \mathrm{s})$, and LogRF power $(3 \mathrm{~W})$, this event can be characterized as a weak IE, similar to the two IC flash IEs described in Marshall et al. (2019) (although therein the IEs were weaker, with VHF powers of $0.09 \mathrm{~W}$ and $0.54 \mathrm{~W}$, and each lacked a coincident E-change pulse). The cumulative light intensity time series (Fig. 3a, b) derived from the full-frame video data shows at most a possible very slight increase starting at the time of the IE. However, this possible increase, essentially a slight rise in background level by $3-5 \%$, is so weak that it is difficult to distinguish above the noise level in the cumulative intensity, and we, therefore, conclude that no significant luminosity was detected with this IE.

The first IB cluster in Example 2 occurs $1.237 \mathrm{~ms}$ after the IE. As in Example 1, the main bipolar E-change pulse may be a classic IBP or it may be a positive NBE. This bipolar pulse is much larger than the IE in E-change and LogRF amplitude and is located within $200 \mathrm{~m}$ of the IE (Fig. 4d). The E-change $E_{100 \mathrm{~km}}$ amplitude $(4.3 \mathrm{~V} / \mathrm{m})$ and LogRF power $(76 \mathrm{~W})$ are the largest of any activity in the IB stage of this flash. This event, expanded in Fig. 3c, can be characterized as a classic IB pulse with subpulses on the leading positive peak of the bipolar waveform (e.g., Weidman and Krider 1979) or it can be characterized as a positive NBE of Type D (Karunarathne et al. 2015; Bandara et al. 2020) waveform, having zero-to-peak risetime of $7.7 \mu$ s and duration of $33.1 \mu \mathrm{s}$ in the E-change data. There are smaller amplitude VHF pulses (some with coincident E-change pulses) during the $78 \mu$ s before the classic IB or NBE; such pulses are rarely seen before an NBE. The E-change pulse marked with the red arrow in Fig. 3a is quite similar, except with less negative overshoot in the E-change signature, to the IE of this flash. In the classic IB or NBE, the VHF peak nearly coincides with the sub-pulse on the rising side of the bipolar waveform that precedes the main E-change peak by $3.2 \mu \mathrm{s}$. We note that if this event is characterized as a classic IB pulse, then it has a larger $E_{100 \mathrm{~km}}$ amplitude and shorter duration than all but two of 40 largest IB pulses in IC flashes studied by Smith et al. (2018). Interpreting this event as the first classic IB pulse yields an Initial E-change (IEC) duration of $1.23 \mathrm{~ms}$, similar to that found for IC flashes in previous studies (Marshall et al. 2014, 2019; Chapman et al. 2017).

As shown (expanded) in Fig. 3b, the cumulative intensity detected within the video frame distinctly increases (from values of $\sim 800$ to peak of 1100 , up to $37 \%$ above background) at the time of this very large event, whether it is an NBE or IBP. The obvious increase begins at the time of the E-change peak (black up-arrow in Fig. 3) and rises to the intensity peak (blue down-arrow, Fig. 3) through about $200 \mu$ s (30 frames). In this case, the luminosity peak coincides approximately in time with the end of significant VHF activity (at about 50.82338-50.82343 s in Fig. 3c). The decrease to near background values of intensity takes about $610 \mu$ s after the peak, during a quiet period in E-change and only weak VHF activity (Fig. 3c), for total enhanced luminosity duration of $0.81 \mathrm{~ms}$.

In the next $1.2 \mathrm{~ms}$, four brief VHF and narrow IB pulses are detected and located nearby (light green circles in Fig. 4, $50.824-8252 \mathrm{~s}$ ), within $200 \mathrm{~m}$ horizontally and slightly higher in altitude than the IE and NBE. The first of these has a relatively large VHF power of $7 \mathrm{~W}$. The last of these narrow pulses is accompanied by a slight intensity increase detected by the camera (Fig. 3a), with a duration of about $320 \mu$ s. The earlier three of these narrow pulses may emit enough detected light to keep the intensity values above background between 50.824 and $50.825 \mathrm{~s}$ (Fig. 3a), but there is not an obvious intensity increase with each pulse. Just after $50.827 \mathrm{~s}$, the second IB cluster (purple arrow in Fig. 3a) occurs with a clearly detected intensity increase, which begins rising near in time to the classic IB pulse peak. This enhanced intensity rises to a peak value $(\sim 900)$ in $0.22 \mathrm{~ms}$, and the enhancement lasts about $0.82 \mathrm{~ms}$. The VHF power with this IBP peaks at $10 \mathrm{~W}$. After this IBP, three more brief VHF and narrow IB pulses are detected and located, between 50.828 and $50.830 \mathrm{~s}$ (red circles in Figs. 3a and 4); the first of these is accompanied by a slight intensity increase which apparently lasts through the time of the second pulse. The last of these small pulses is closest to the camera but does not cause an appreciable increase in the cumulative intensity.

For $140 \mathrm{~ms}$ after the time shown in Fig. 4, the Example 2 IC flash continued developing to the south and east, at $8.0-10.5 \mathrm{~km}$ altitude; most this activity was not visible in the camera data (due partly to the location in greater reflectivity and partly to the location above the image). At $50.968 \mathrm{~s} \mathrm{a}$ long period of intermittent visible activity developed $1.5 \mathrm{~km}$ 
Flash Time: 04:43:50.820 - 50.830 UT on 22-Jun-2019 Radar Time: 04:41:58

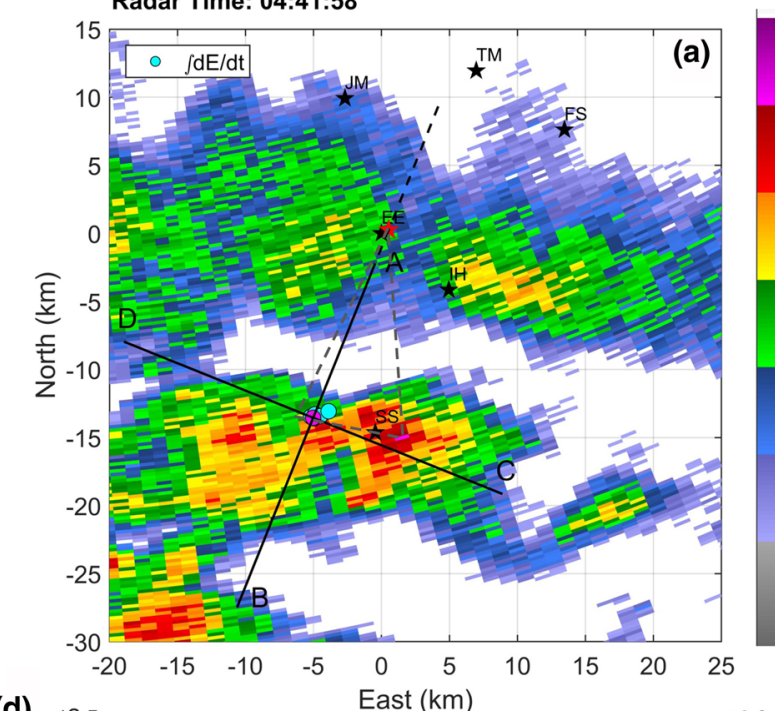

(d)

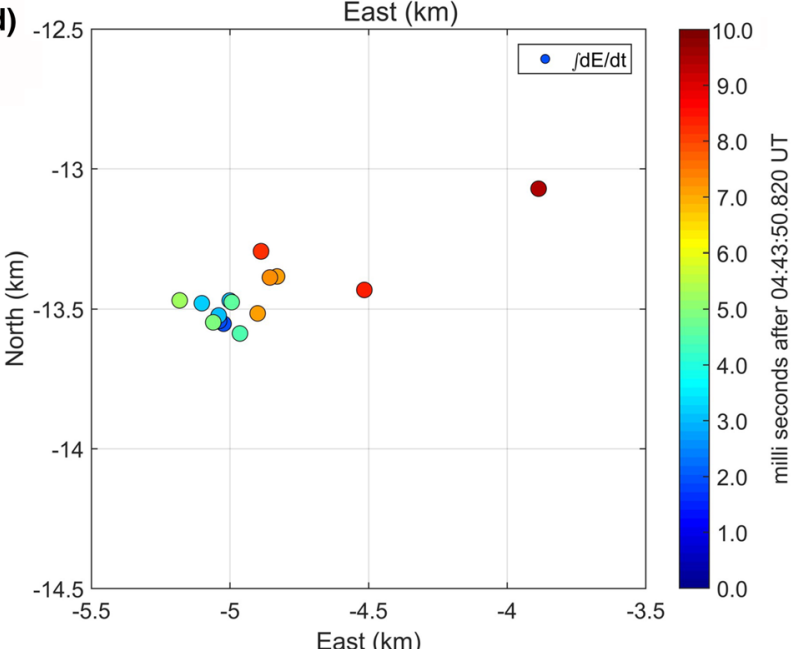

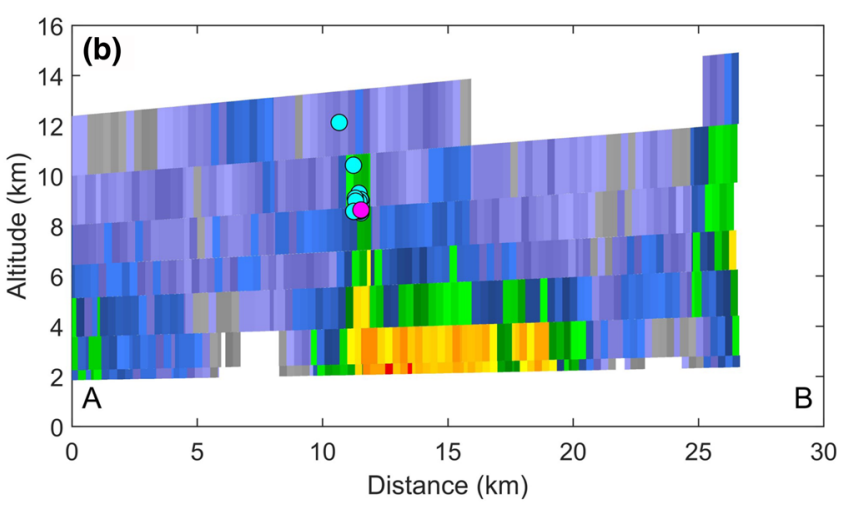

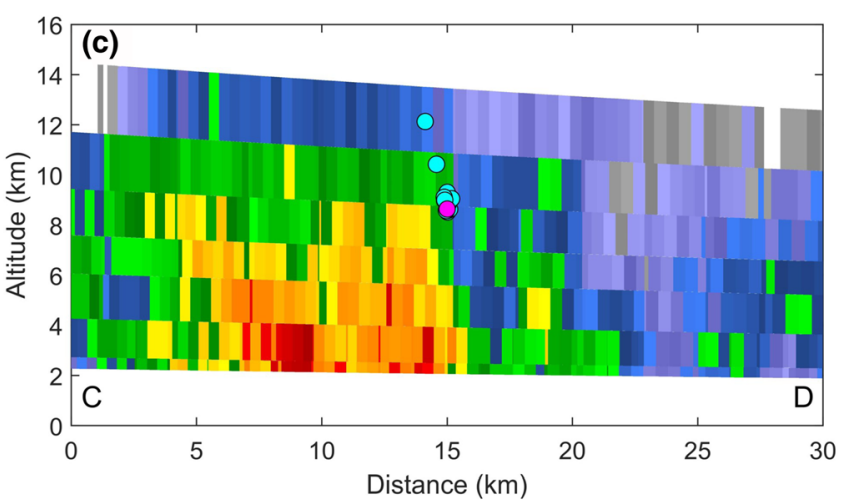

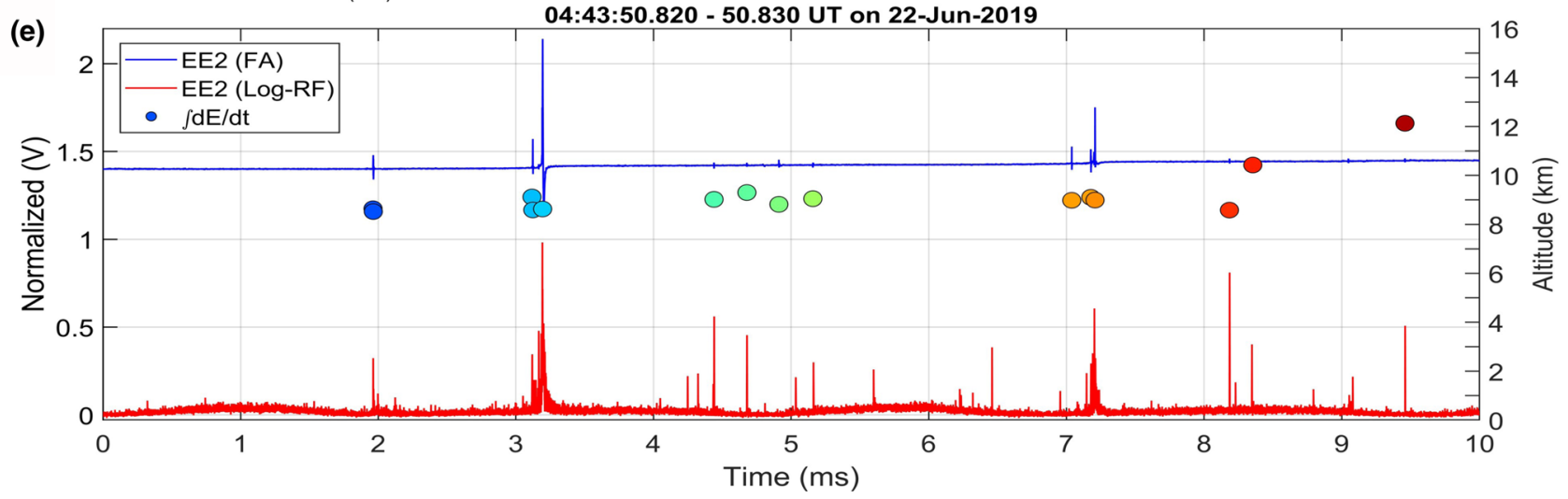

Fig. 4 Radar reflectivity and pulse locations for Example 2, IC flash at 0443:50 UT on 22 June 2019. a Plan view of low-level PPI scan, with locations of pulses for the first $10 \mathrm{~ms}$ of the flash overlaid. (First pulse is magenta, others are cyan.) Black stars are electromagnetic sensor sites, red star is camera location (with field-of-view indicated by dashed lines), and black lines indicate cross-section locations. b
Radar cross-section along line of sight of the camera toward the start of IC flash. c Radar cross-section perpendicular to $\mathbf{b}$ and approximately aligned with video camera image. d Plan view of first $10 \mathrm{~ms}$ of pulse locations, color-coded by time, for the expanded area of initiation. e Time series of normalized E-change (FA), VHF (logRF), and pulse altitudes (color-coded as in d) for first $10 \mathrm{~ms}$ of flash 

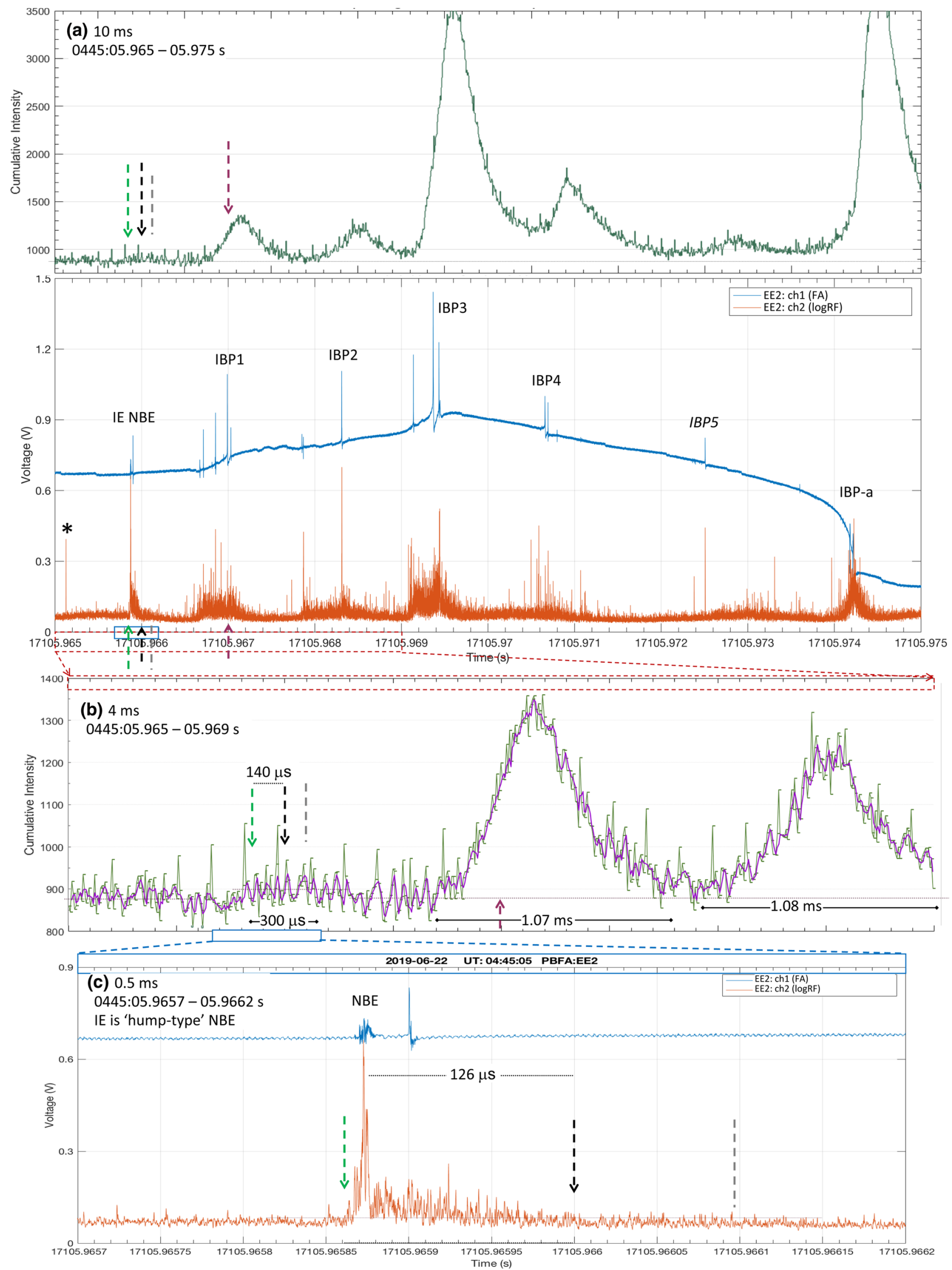
4Fig. 5 Similar to Fig. 3 except showing beginning of Example 3 IC flash at 0445:05 UT on 22 June 2019, approximately $17 \mathrm{~km}$ slant range from camera. a First $10 \mathrm{~ms}$ of E-change (FA) and VHF (logRF) data, with cumulative intensity data at top. Green arrow indicates beginning of the initiating event (IE), a positive NBE, and black arrow $140 \mu$ s later is the end time of strong VHF emissions associated with the IE. Gray vertical line indicates the approximate end of VHF activity above background level. Purple arrow marks the classic IBP of the first IB cluster (IBP1); other IB clusters are labeled. Red and blue rectangles indicate times in lower figure parts. The asterisk $(*)$ denotes a spike detected only at the EE sensor, assumed due to either noise or a distant event. b Cumulative intensity data for $4 \mathrm{~ms}$. A slight increase in intensity (average 3\%, peak 7\% above background) from background average (dashed horizontal) is detected during the IE (NBE), and larger intensity increases accompany the two IB clusters. Durations are given for first two IB clusters' intensity enhancements (time above background, peaks are 55\% and $45 \%$ above background). c FA and $\log R F$ data for the first $500 \mu \mathrm{s}$, which show the IE was a positive 'hump-type' NBE. Dotted horizontal line within the $\operatorname{logRF}$ data indicates the approximate background (upper level of noise)

to the north, nearer to the edge of the storm. A topic for another study is the leader propagation that is visible in the video data by $51.0286 \mathrm{~s}$; that activity resulted in several extensive IC strokes starting $150 \mathrm{~ms}$ later, with visibly bright luminosity through durations in excess of $1.5 \mathrm{~ms}$. The final IC stroke of the flash occurred at 0443:51.537 UT.

\subsection{Example 3}

Data for the beginning of our third example, an IC flash at 0445:05 UT on 22 June 2019, are shown in Fig. 5. Locations for this flash were not available from PBFA because only three sites had triggered data. However, the camera orientation did not change after the previous flashes, including Example 2, $75 \mathrm{~s}$ earlier. Thus, we can estimate this flash initiation location from its relative placement on the video frame. Figure 6 shows the approximate location, at $14.6 \mathrm{~km}$ distance from the camera, or $16.8 \mathrm{~km}$ slant range (using estimated altitude of $8.6 \mathrm{~km}$, from Example 2). This estimate fits well with the first LINET location for the flash, coincident with an IB pulse (IBP-a, shown in Fig. 5) that was $8.2 \mathrm{~ms}$ after the IE but much higher, at $12.2 \mathrm{~km}$ altitude. Corresponding imaged area at $16.8 \mathrm{~km}$ slant range is $8.6 \mathrm{~km} \times 11.5 \mathrm{~km}$; at the slant range to the known LINET location, the imaged area is $10.0 \mathrm{~km}$ across $\times 13.4 \mathrm{~km}$ top altitude. The radar reflectivity data in Fig. 6 indicate this IC flash initiation was beside the storm core, with echo tops in the vicinity at $12-14 \mathrm{~km}$ altitude.

The cumulative intensity data in Fig. 5a for the first $10 \mathrm{~ms}$ of Example 3 show seven intervals of increased luminosity detected by the video camera. The first of these increases corresponds in time with the IE, while the next six increases are coincident with the six labelled IB clusters, as detected by the VHF and E-change sensors.
The first light increase is weak, with a maximum about 5-7\% above background intensity values (Fig. 5b), and it is coincident with the IE, which in this flash was a low-power, positive NBE (Fig. 5c). The E-change data for this NBE do not resemble any of the common types described by Karunarathne et al. (2015) or Leal et al. (2019). The relatively small E-change amplitude and lack of significant overshoot peak of this positive NBE are similar in shape to the "more monopolar" NBEs of both polarities studied by Rison et al. (2016) and to the opposite polarity, 'hump-type' negative NBEs studied by Bandara et al. (2019). Using the estimated slant range to the IE $(16.3 \mathrm{~km})$, the NBE has a VHF power of $43 \mathrm{~W}$, an E-change $E_{100 \mathrm{~km}}=0.30 \mathrm{~V} / \mathrm{m}$, and an E-change duration of $12 \mu$ s. About $50 \mu \mathrm{s}$ after the NBE, a narrow bipolar IBP occurs with a VHF power of $1 \mathrm{~W}, E_{100 \mathrm{~km}}=0.72 \mathrm{~V} / \mathrm{m}$, and an E-change duration of $5 \mu \mathrm{s}$. Although the NBE power of $43 \mathrm{~W}$ is relatively weak for NBEs, this IE can be considered as a strong IE compared to IEs of two IC flashes described by Marshall et al. (2019) with VHF powers $<1 \mathrm{~W}$.

To our knowledge, these are the first published data showing visible light detected with a positive NBE initiating an IC flash. For the IE the video intensity values begin to increase close to the time of the initial VHF emissions (green arrow, Fig. 5), and the peak in intensity occurs about at the time when the VHF emission drops to smaller values (black arrow, Fig. 5). In the intervening $140 \mu$ s of appreciable VHF emission, when the average detected light in the camera frame is increasing, the VHF and E-change pulse peaks of the NBE occur. The duration of the 'shoulder' in VHF emissions after the VHF peak, and the interval between VHF peak and light intensity peak, are both about $126 \mu$ s. A period of VHF activity that is above background level extends for about $95 \mu$ s, yielding a total VHF duration of about $235 \mu \mathrm{s}$. This duration of VHF emission matches reasonably well with the duration of weakly enhanced luminosity, about $300 \mu \mathrm{s}$, for the IE (NBE).

After the IE and for the remaining $8.5 \mathrm{~ms}$ of the record that is included in Fig. 5, each subsequent luminosity increase is coincident with an IB cluster. A longer portion of the IB stage of the Example 3 flash is shown in Fig. 7. This $50 \mathrm{~ms}$ period includes the IE and 10 large increases in light intensity that are associated with 10 IB clusters. Data for the periods surrounding nine of these IB clusters are expanded in Figs. 8, 9 and 10.

The general character of this IB stage can be described based on Figs. 8, 9 and 10 and the quantitative parameters listed in Table 1. In each IB cluster, the enhanced luminosity increase is marked with a horizontal black line with diamonds at each end; the beginning of the increase occurs within $0-120 \mu$ s after the start of appreciable and continuous VHF emission (marked with a red vertical arrow). The 
onset of significant continuous VHF emission in each event is $90-520 \mu$ s before the main positive E-change peak of the largest classic IBP in the IB cluster (marked with purple dashed arrow). (Note that the classic IBP of the IB cluster labeled IBP-bb has the smallest E-change amplitude and the shortest duration (11 $\mu \mathrm{s})$ in all the clusters, which may be related to its relatively weak luminosity.) In IBP-d (Fig. 10b), there is also intermittent VHF in three short bursts during the $800 \mu$ s preceding the main pulse. Eight of the ten IB clusters exhibit 1-5 narrow IB pulses (smaller E-change pulses) during the period of large VHF emissions that precedes the largest classic IB pulse. The largest E-change peak is first in IBP4 (Fig. 8b), while IBP-bb (Fig. 9b) has only one large E-change pulse. In half the clusters (IBP2, IBP5, IBP-bb, IBP-b, and IBP-d) the largest VHF peak and largest E-change peak coincide, to within $1 \mu$ s. In the other five clusters, the VHF peak occurs $80-150 \mu \mathrm{s}$ before (IBP4, IBP1) or 5-60 $\mu$ s after (IBP-a, IBP-c, IBP3) the largest E-change peak. The duration of significant VHF activity with each cluster is about 700-900 $\mu$ s, except in IBP-d where it is only about $250 \mu$ s. As seen in Figs. 8, 9 and 10, the strongest VHF activity in each IBP event has approximately the same duration as the luminosity increase.

In addition to being close in time to the onset of VHF emission, the time of the beginning of detected luminosity increase is typically accompanied by a noticeable E-change pulse; IBP-a and IBP-d are the exceptions to this. A steeper rise of enhanced luminosity, i.e., an inflection point on the cumulative intensity increase, occurs in four IB clusters (IBP-a, IBP-b, IBP-c, IBP-d) at the time of a large classic IB pulse that is also coincident with the peak of VHF emission. Luminosity detected with other IB clusters manifests as a steady rise to the peak intensity, and in those cases, the largest E-change pulse is not coincident with the largest VHF emission. In nearly all IB clusters, the intensity peak is reached (i.e., intensity increase stops) near the time when the large VHF emissions cease; IBP-bb (Fig. 9b) is an exception to this, as the intensity peak in this relatively weak event is reached about $160 \mu$ s later. The time of the intensity peak in all these IB clusters is also usually coincident with when the E-change data stop oscillating, particularly in the later IB clusters (IBP-b, IBP-c, IBP-d) which have a longer duration of E-change variations. These later IB clusters also have a series of rapid, small E-change oscillations leading up to each IBP, which we discuss later with regard to the mechanism of Kostinskiy et al. (2020).

All 10 of the IB clusters shown for Example 3 (Figs. 7, 8, 9, 10) have appreciable VHF emission during the decrease of light intensity. Hence, it seems the period after the peak intensity is not only fading, scattered light detected by the camera; rather, there is apparently weak light emission during these extended times of VHF activity. A slower fall-off of intensity and longer duration of intensity enhancement is observed in IB clusters with more VHF continuing after the intensity peak (e.g., IBP-a vs. IBP-b, and IBP-c vs. IBP-d). Related to this, it is generally the case for this flash that the earlier IB clusters (IBP1 to IBP5) have cumulative intensity increases that are more symmetric about their peak (nearly equal rise and fall) compared to later IB clusters (IBP-a to IBP-d), which show a rise to peak cumulative intensities in less time than they return to near background intensities. Later IB clusters also have slower intensity decreases than the early IB clusters and generally longer overall luminosity durations, along with more continuing VHF activity after the E-change variation of the IBP in the FA data. The average duration of enhanced luminosity in these 10 IB clusters is $1.26 \mathrm{~ms}$ (Table 1 ).

Throughout the first $50 \mathrm{~ms}$ of this IC flash, very brief bursts of VHF (with or without a narrow IB pulse) occur which show little or no concurrent light intensity increase (e.g., between IBP1 and IBP2, between IBP3 and IBP4, between IBP-bb and IBP-b), at least in the cumulative values. However, these brief bursts of VHF emission may be having a weak effect on luminosity which is detected as a slightly higher background cumulative intensity throughout these periods. Since the events are within the cloud, we do not know but can expect these brief bursts may be producing light in very small areas.

Two of the IB clusters in this flash deserve additional mention. First, we note that the classic IB pulse in IBP2 (Fig. 7b) also has the character of a positive NBE, with Type A waveform per Karunarathne et al. (2015). This possible NBE has a duration of $16 \mu \mathrm{s}$, and it exhibits a larger E-change amplitude and stronger VHF peak power than the IE of this flash. Based on the estimated range to the event, the VHF source power of IBP2 is about $55 \mathrm{~W}$ and $E_{100 \mathrm{~km}}$ is $1.5 \mathrm{~V} / \mathrm{m}$. The VHF power would make this a relatively weak NBE or a strong classic IB pulse, while the $E_{100 \mathrm{~km}}$ amplitude is relatively small for either type of event. As described above, the character of the luminosity detected during IBP2 is similar to the other IB clusters in this series. Notably, however, the luminosity increase does not appear to change its slope at the time of the possible NBE, and the peak intensity is weaker than most of the events (ranked eighth of ten). Second, we mention that IB cluster IBP-a (Fig. 7d) is the first event of this flash located by LINET, at $12.2 \mathrm{~km}$ altitude, with $4.8 \mathrm{kA}$ estimated peak current. IBP-a has a large classic IB pulse at EE (and is within its reversal distance of $17 \mathrm{~km}$ ). Its altitude, much higher than that estimated for the IE, suggests this flash may have behaved similarly to Example 2 by jumping upward in altitude by about $3.5 \mathrm{~km}$ within the first $9 \mathrm{~ms}$ its IB stage. In terms of the peak cumulative intensity value, IBP-a is the brightest IB cluster during the first $50 \mathrm{~ms}$, although neither its VHF peak power $(12 \mathrm{~W})$ nor its $E_{100 \mathrm{~km}}$ amplitude $(1.9 \mathrm{~V} / \mathrm{m})$ are especially large in comparison to the surrounding events in time. 
(a) base scan PPI, 2019-06-22 0446:25 UT

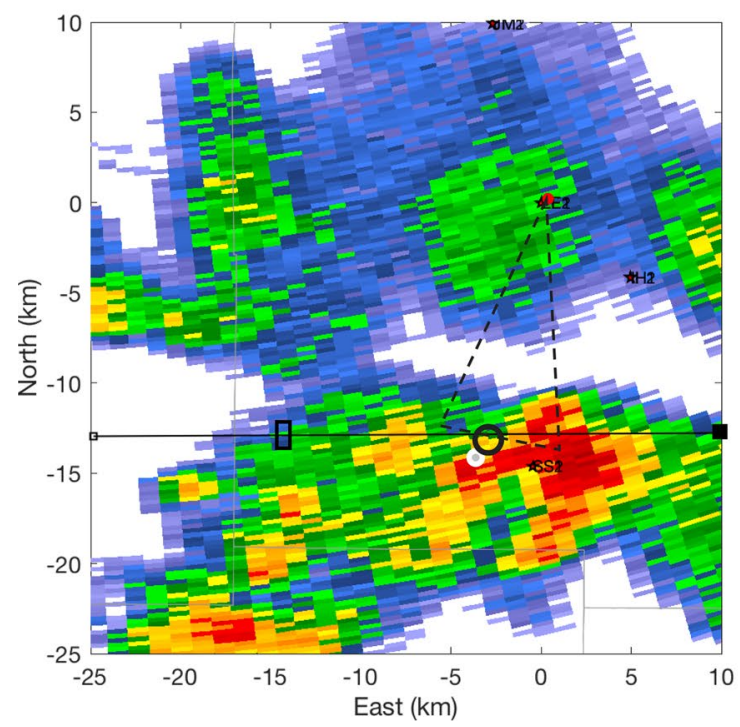

(b) west-to-east cross-section

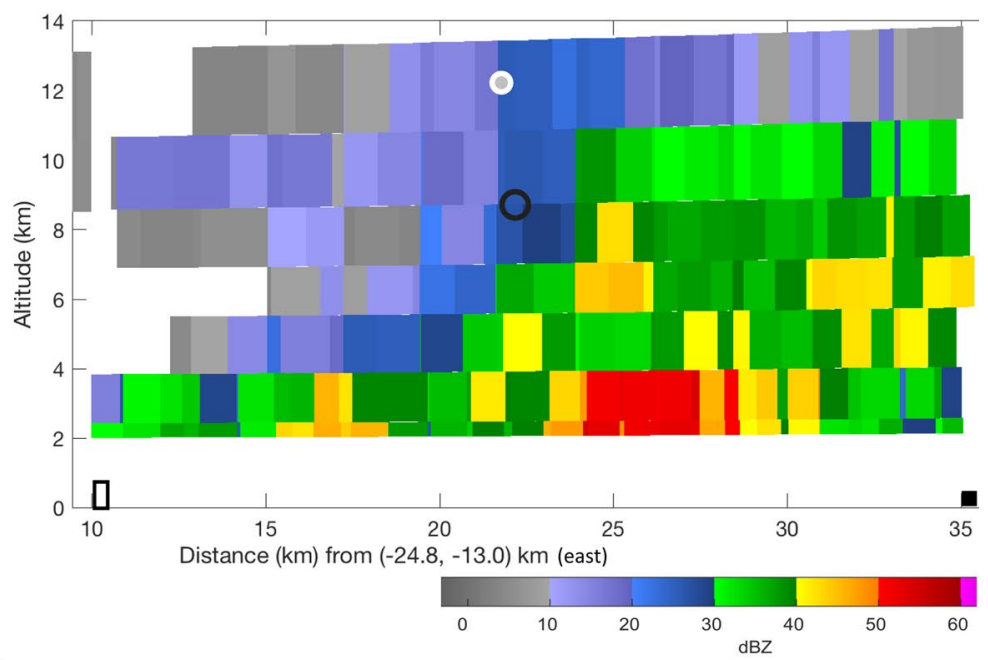

Fig. 6 Radar reflectivity for Example 3, IC flash at 0445:05 UT on 22 June 2019. a Plan view of low-level scan, including locations of camera site (red dot), camera field-of-view (dashed cone), approximate flash beginning (black circle), and first LINET source (white circle) of IBP-a, $8.2 \mathrm{~ms}$ after the initiating NBE. Black line is cross-section location. b Radar cross-section through storm, near location of IC flash beginning, with first LINET and approximate initiation locations projected

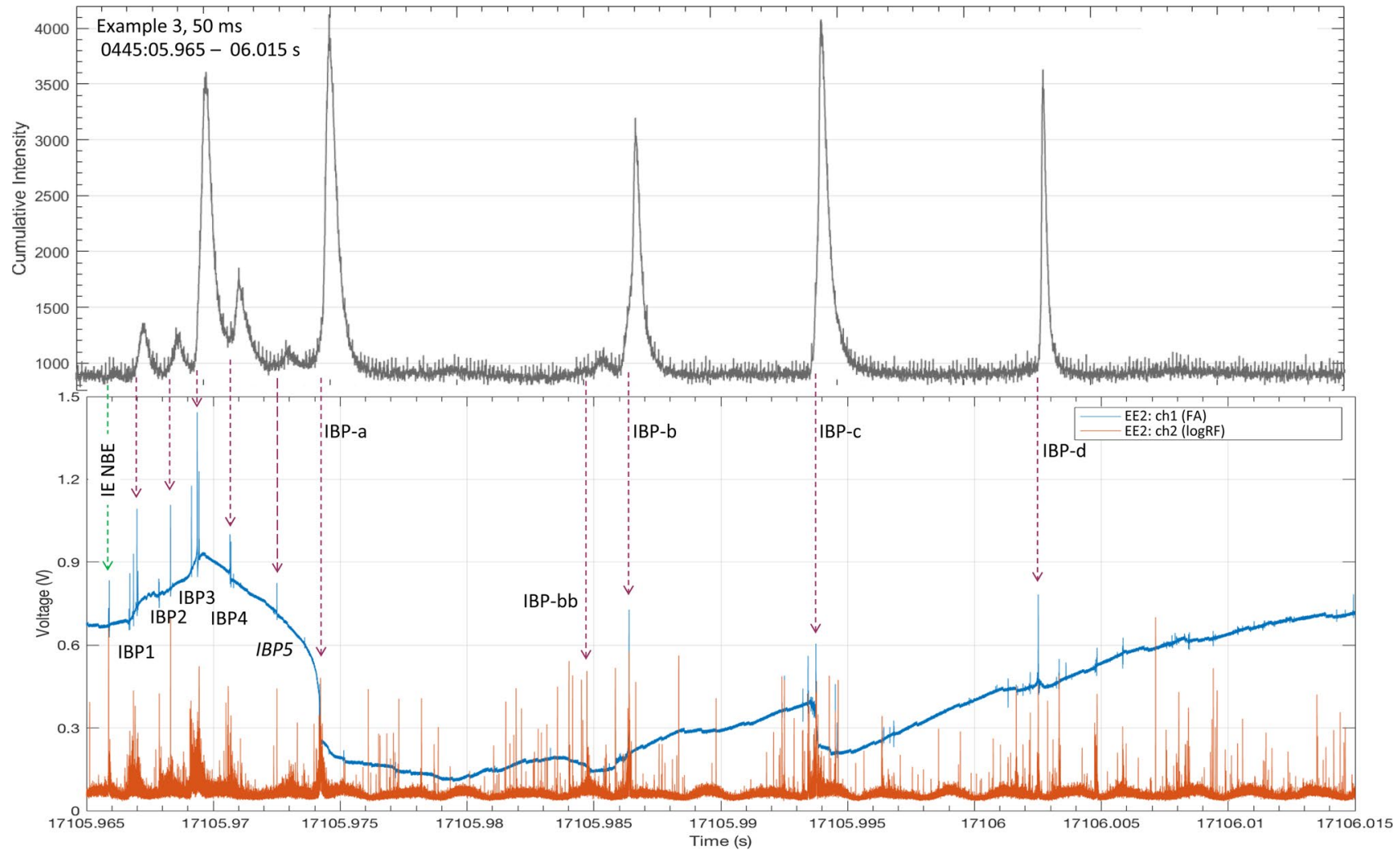

Fig. 7 Beginning and IB stage of IC lightning flash Example 3 at 0445:05 UT (17,105 s past 00 UT) on 22 June 2019, approximately $17 \mathrm{~km}$ slant range from camera. Presentation is as in Fig. 5 except showing first $50 \mathrm{~ms}$ of cumulative intensity, E-change (ch1, FA) and VHF (ch2, logRF) data. Green arrow indicates initiating event (IE), a positive NBE. Purple arrows are the largest classic IBP in each of the ten labeled IB clusters. Exposure time per video frame is $6.1 \mu$ s. (Offset of time axes is in the axes, only; data curves are vertically timealigned, and camera data are approximately adjusted to light arrival at $\mathrm{EE}$ site.) 

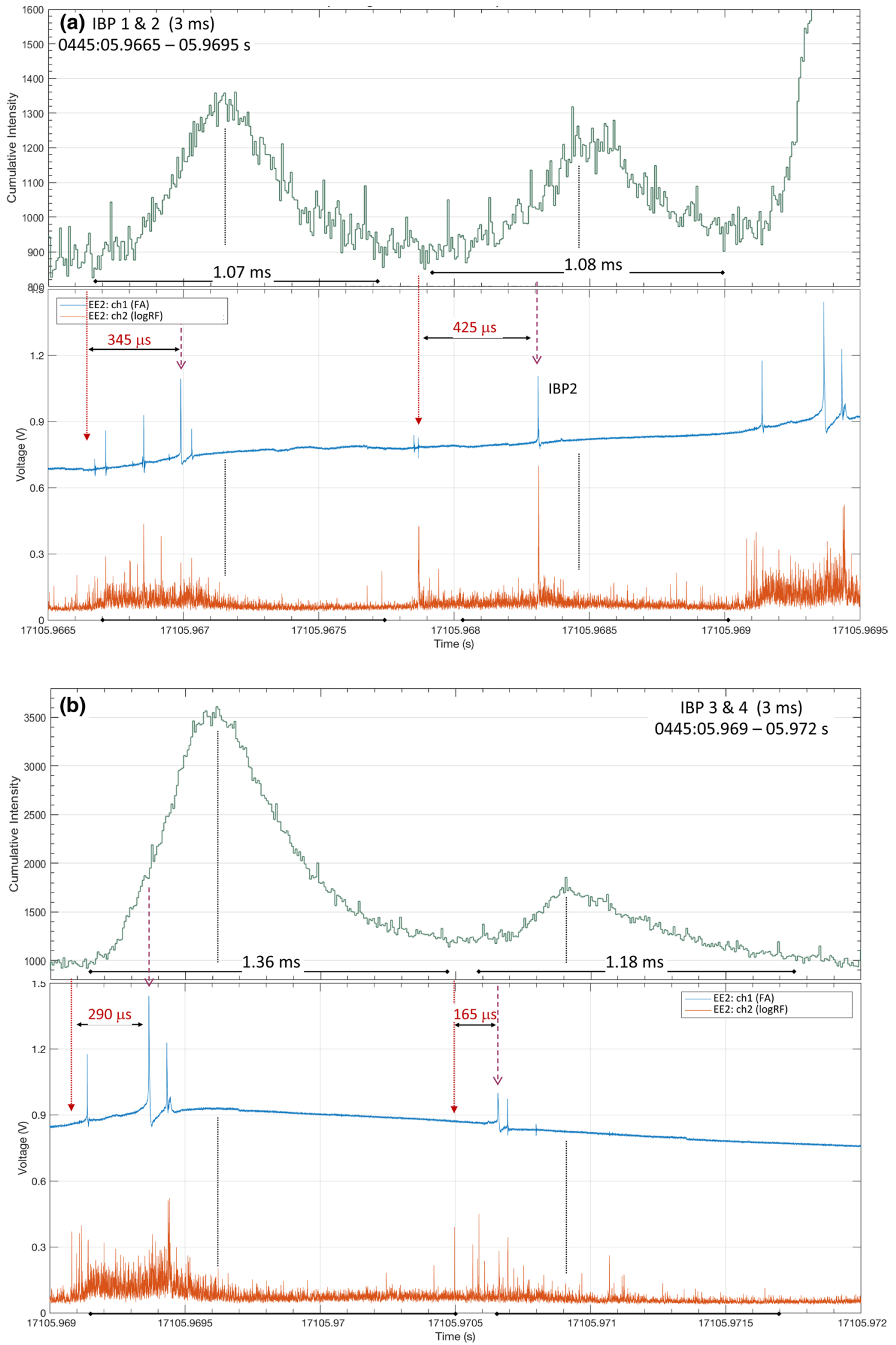
4Fig. 8 a Expanded data for IB clusters IBP1 and IBP2, as labeled in Fig. 7. IBP2 has estimated VHF source power of $55 \mathrm{~W}$, and the simple bipolar E-change waveform is either a classic IBP or a Type A positive NBE. b Expanded IB clusters IBP3 and IBP4, as labeled in Fig. 7. The duration of enhanced luminosity for each IB cluster is indicated on the cumulative intensity plot and on the time axis (horizontal bar), and the dotted vertical bar indicates the time of peak intensity during each IB light burst. Purple arrows indicate time of each peak in E-change data. Red arrows indicate onset of large VHF emission of each cluster

Later data not shown for this flash indicate the IB stage continued for another $100 \mathrm{~ms}$, with weaker luminosity detected intermittently (until 0445:06.1160 UT). After this stage, distinct channel was intermittently visible out the top of the cloud, starting at 0445:06.131314 UT. Flash activity was very visible at intervals for $265.65 \mathrm{~ms}$ (through about 0445:06.396963). The second LINET-detected event in this flash was $242.3 \mathrm{~ms}$ after the first (IBP-a), at $11.0 \mathrm{~km}$ altitude, coincident with an IC stroke (at 0445:06.216515 UT) seen well by the camera. The later IC leader activity and IC strokes in this flash are subjects of a separate study.

\subsection{Example 4}

The fourth case of luminosity data during the beginning of an IC flash is shown in Fig. 11. This flash occurred at 0451:34 UT on 22 June 2019, about 6.5 and 7.8 min later and on the opposite (leading) side of the same storm as Examples 2 and 3. Radar data and pulse locations for the first $10 \mathrm{~ms}$ are shown in Fig. 12. The IE in this case is a simple 'Type A' NBE (Karunarathne et al. 2015). It was located at $7.85 \mathrm{~km}$ altitude and $18.2 \mathrm{~km}$ distance from the EE sensor site. The VHF power estimate is $990 \mathrm{~W}(29.9 \mathrm{dBW})$, within range of values $(0.18-88.4 \mathrm{~kW})$ given by Bandara et al. (2020) for positive NBEs that initiated IC flashes in this same geographical region. The range-normalized E-change amplitude, $E_{100 \mathrm{~km}}$, is $3.7 \mathrm{~V} / \mathrm{m}$, which is large compared to the average for 3 Type A NBEs $(2.0 \mathrm{~V} / \mathrm{m})$ found by Bandara et al. (2020) and large compared to the average of the largest classic IB pulse in $40 \mathrm{IC}$ flashes $(1.49 \mathrm{~V} / \mathrm{m})$ found by Smith et al. (2018). After the IE, the locations for the IB pulses and other small events (Fig. 12) show that the flash propagated upward to $10.8 \mathrm{~km}$ altitude within the first $10 \mathrm{~ms}$.

Cumulative intensity data (Fig. 11a, b) are noisy but indicate a possible weak enhancement for about $350 \mu$ s after the IE with average intensity rising to $<5 \%$ above background values. The radar and source position data in Fig. 12 show the events were slightly lower and much deeper within the cloud, relative to the camera, compared to Examples 2 and 3 (Figs. 4 and 6). In this case, any light emitted during the earliest pulses would travel through at least $10 \mathrm{~km}$ of $\geq 30 \mathrm{dBZ}$ reflectivity to get to the camera site. This difference in visibility conditions may explain the weaker luminosity detected by the camera during this stronger event. The cloud conditions near the initiation may also play a role in the emission and detectability of light: in Example 4 the nearby echo top had fallen to $\sim 12 \mathrm{~km}$ altitude (Fig. 12b), compared to 9 min earlier (Fig. 4c) when the area had 20-30 dBZ echo to at least $13 \mathrm{~km}$ altitude.

The early IB pulses, beginning about $3.2 \mathrm{~ms}$ after the IE, were successively higher in the cloud. The series of IB pulses comprising the first two IB clusters show obvious but small intensity increases of 6-12\% above background values. These two IB clusters included in Fig. 11a have character similar to those detailed for Example 3 (Figs. 8, 9, 10). In particular, the largest classic IB pulse and VHF emission peak in each occur during the rise in cumulative intensity, and the peak cumulative intensity occurs as the largest VHF emissions cease. Weaker VHF emission continues and a few smaller amplitude IB pulses occur through the time of the fall-off in cumulative intensity in both IB clusters shown in Fig. 11a. Total durations of the enhanced intensity are 1.44 and $1.65 \mathrm{~ms}$ with these IB clusters. Prior to the first of these IB clusters, there is a series of narrow IB pulses in the E-change and LogRF data which are accompanied by weak luminosity, lasting about $800 \mu$ s and similar to the light detected during the IE.

After the first $10 \mathrm{~ms}$ of this flash, there was very little luminosity detected for the next $\sim 108 \mathrm{~ms}$, at which time flash activity (not shown) re-started near the same location as the IE. For the next $127 \mathrm{~ms}$, events in this IC flash had diffuse luminosity detected at intervals, and then leaders, channels, and IC strokes became clearly visible intermittently through and out the top of the cloud for another $232 \mathrm{~ms}$. The flash activity ended at 0451:34.834 UT, about $477 \mathrm{~ms}$ after the IE.

\subsection{Example 5}

The last flash we consider, Example 5, occurred at 0452:27 UT in same storm as Examples 2, 3 and 4. Relative to Example 4, this IE was $53 \mathrm{~s}$ later than and $6.7 \mathrm{~km}$ west-southwest ( $x=4.8 \mathrm{~km}, y=-17.7 \mathrm{~km}$, marked in Fig. 12a). As shown in Fig. 13, altitude of initiation was $7.69 \mathrm{~km}$, and the early IB pulses indicate the flash ascended to $11 \mathrm{~km}$ within the first $10 \mathrm{~ms}$. As with Example 4, location data for Example 5 indicate this IE is relatively obscured, on the far side of the convective cell relative to the camera site, at $20.4 \mathrm{~km}$ slant range.

We include this example primarily because the cumulative intensity during the IE, a weak event, responds similarly to that during the IE of Example 4, a NBE. Figure 13 shows the time series of E-change, VHF, and intensity data for Example 5. The IE is a weak VHF pulse (at 17,547.37083 s) with a power of $1 \mathrm{~W}$ and a duration of $1.8 \mu \mathrm{s}$; there is no accompanying E-change pulse. Cumulative intensity is $\sim 3 \%$ 
above background levels beginning about $50 \mu$ s after the weak IE pulse and continuing for about $520 \mu \mathrm{s}$. After the IE there are five small narrow IB pulses before the first classic IBP; two of these had coincident weak VHF pulses. The first three narrow IBPs are within the duration of enhanced intensity that had started after the IE, and these IBPs may have also increased the light intensity.

The three IB clusters in the first $10 \mathrm{~ms}$ of Example 5 (Fig. 13a) are similar to the IB stage observations in the other flashes presented herein. The first IB cluster has two classic IB pulses; the first of these has a complicated E-change waveform $\left(E_{100 \mathrm{~km}}\right.$ of $\left.3.0 \mathrm{~V} / \mathrm{m}\right)$ with substantial VHF emission (peak value of $4.4 \mathrm{~W}$ ) for at least $200 \mu \mathrm{s}$, and enhanced intensity detected by the camera for about $1.12 \mathrm{~ms}$ duration (Fig. 13b. The intensity increase begins about at the time of the E-change and VHF peaks, and the intensity maximum occurs about $400 \mu$ s later. The other two IB clusters shown (Fig. 13a) have longer overall durations, and each includes two classic IB pulses. In these, the largest E-change and VHF peaks occur during the steep rise in cumulative intensity. Maximum intensity, $14-38 \%$ above background, occurs close to when the substantial VHF emissions cease. Durations of enhanced intensity are 1.20 and $1.67 \mathrm{~ms}$. The last classic IB pulse in this $10 \mathrm{~ms}$ is located near $10.2 \mathrm{~km}$ altitude. However, this flash remained largely obscured by the intervening dense cloud echo relative to the camera. Activity throughout the next 288 ms was intermittently bright but diffuse in the video data.

\subsection{Summary of observations}

In Example 1 (Fig. 2) the weak IE may have caused a slight light intensity increase of 5\% above background intensity, but there is uncertainty that the observed slight increase was caused by the IE. The first IB cluster had a large amplitude E-change pulse that was either a classic IBP or a Type 11 CID (NBE); this E-change pulse was coincident with a modest rise of video-detected light intensity ( $11 \%$ above background). The video intensity peak occurs about $140 \mu$ s (21 frames) after the largest E-change and VHF peaks, near the time of the last IBP in the cluster. The overall duration of the enhanced cumulative intensity with the first IB cluster is about $520 \mu \mathrm{s}$. Much weaker luminosity was detected with several subsequent IB clusters (through the first $13.5 \mathrm{~ms}$ of the IB stage), interpreted as above the camera frame or more heavily obscured in cloud.

The weak IE in Example 2 (Fig. 3) has a small amplitude and is not coincident with significantly detected luminosity above the noisy background intensity. The first IB cluster occurred $1.23 \mathrm{~ms}$ after the IE and included a strong VHF pulse associated with an E-change waveform that could be either a classic IBP or a Type D NBE. The first IB cluster is accompanied by clearly detected luminosity. The obvious cumulative intensity increase begins near the time of the E-change and VHF peaks, and its duration (above background intensity) is about $811 \mu \mathrm{s}$ (122 frames) similar to the duration of VHF emissions. One other large-amplitude IBP and three of the seven narrow IBPs in the first $10 \mathrm{~ms}$ of this flash are also accompanied by luminosity increases that are relatively weak in the detected cumulative intensity data.

Example 3 data (Figs. 5, 6, 7, 8, 9, 10) include evidence of a slight but obvious increase in luminosity associated with the IE. This first event, a weak NBE, is the first known case of light detected coincident with a positive NBE that is an IE. A narrow IBP occurs $50 \mu$ s after the NBE and substantial VHF emissions occur for $126 \mu$ s after the main VHF peak of the NBE. The cumulative intensity in the video frame increases starting near the onset time of the NBE's VHF emissions, it rises weakly through the times of the VHF and E-change peaks, and the intensity peak coincides with the end of VHF emissions associated with the NBE. The duration of large VHF emissions and increasing luminosity is about $140 \mu \mathrm{s}$, and the entire duration of enhanced luminosity with some VHF activity is about $300 \mu \mathrm{s}$. After the IE, through the first $50 \mathrm{~ms}$ of the IB stage, there is appreciable light detected with all the IB clusters; cumulative intensity in the video frame has an average duration of $1.26 \mathrm{~ms}$ for ten complicated IB clusters. The IB clusters, each comprised of one to five pulses in $<750 \mu \mathrm{s}$, show the onset of the intensity increase occurs near or just after the E-change and VHF data begin to vary; the largest pulses in the E-change and VHF data occur during the intensity increase. Appreciable VHF activity continues for a few hundred microseconds as the cumulative intensity values return to background level in each IB cluster. We note that the luminosity of the IB clusters lasted longer than the luminosity durations of $300-800 \mu \mathrm{s}$ found for individual IC-type IB pulses studied by Stolzenburg et al. (2016) with a less sensitive high-speed video camera. These are also longer than the luminosity duration (full width at half maximum) average of $176 \mu$ s found by Wilkes et al. (2016) for individual IC IB pulses using an all-sky photodiode system. This flash was the most visible of those presented and later was observed to extend out the cloud top.

In Example 4 (Fig. 11), the large amplitude, strong IE is a Type A NBE and is coincident with only very weak detected light intensity ( $<5 \%$ above background). Radar and pulse location data indicate this event may be more obscured than the IEs and NBEs in flash Examples 1-3, and this fact may explain the weaker light observed. A series of narrow IBPs before the first IB cluster has weak coincident light. Obvious increases in cumulative intensity are observed with the two IB clusters in the first $10 \mathrm{~ms}$, with increases beginning just before the largest E-change and VHF peaks and lasting above background for about 

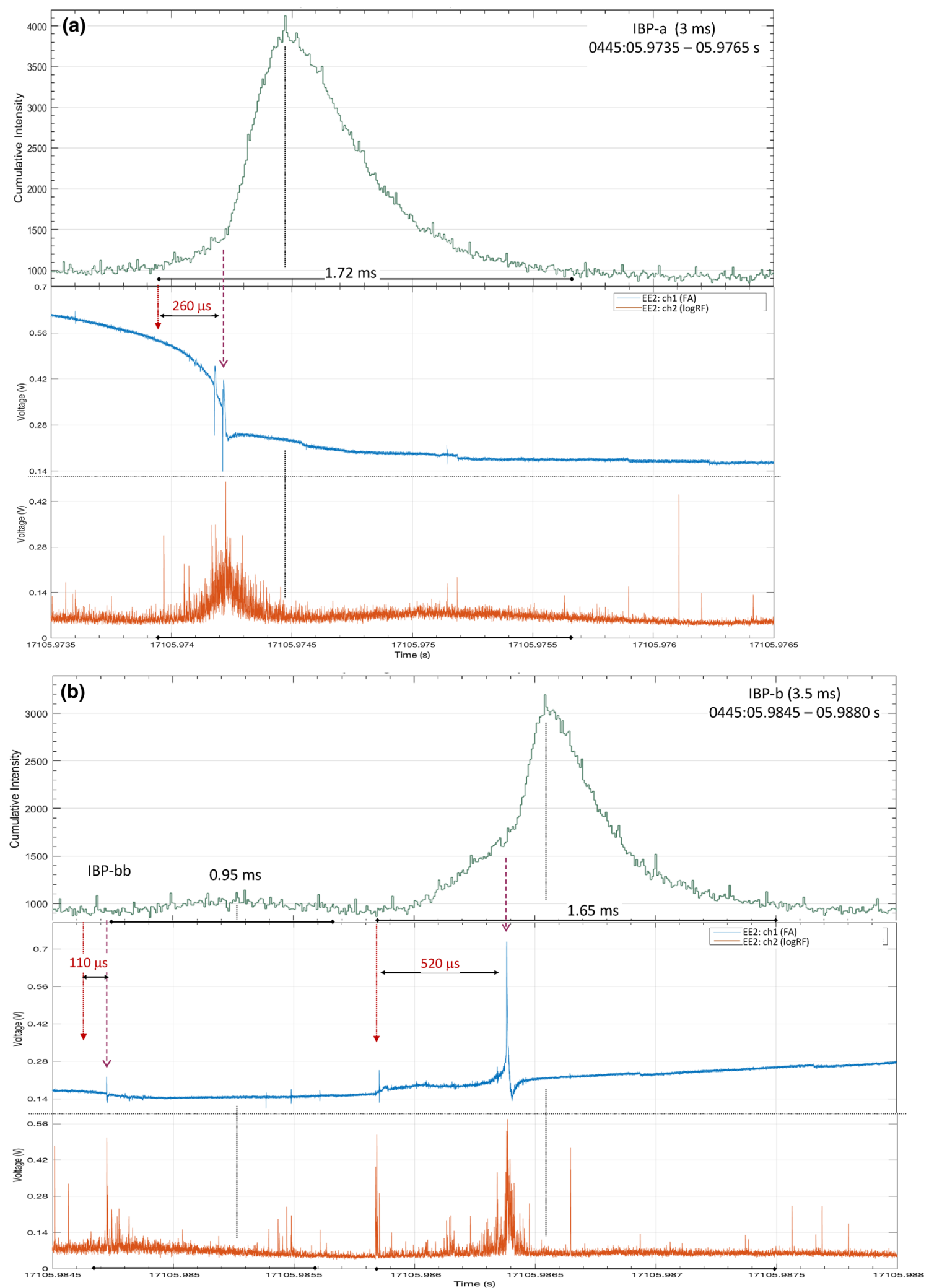

Fig. 9 a Data for IB cluster IBP-a, as labeled in Fig. 7, expanded. The main peak of this cluster was located by LINET at $(x=-3.4$, $y=-14.6, z=12.2) \mathrm{km}$, within the reversal distance of the EE sensor. b IBP-bb and IBP-b, as labeled in Fig. 7, expanded. The duration of enhanced luminosity for each IB cluster is indicated on the cumula- tive intensity plot and on the time axis (horizontal bar), and the dotted vertical bar indicates the time of peak intensity during each IB light burst. Purple arrows are peak times in E-change data. Red arrows indicate onset of large VHF emission of each cluster 

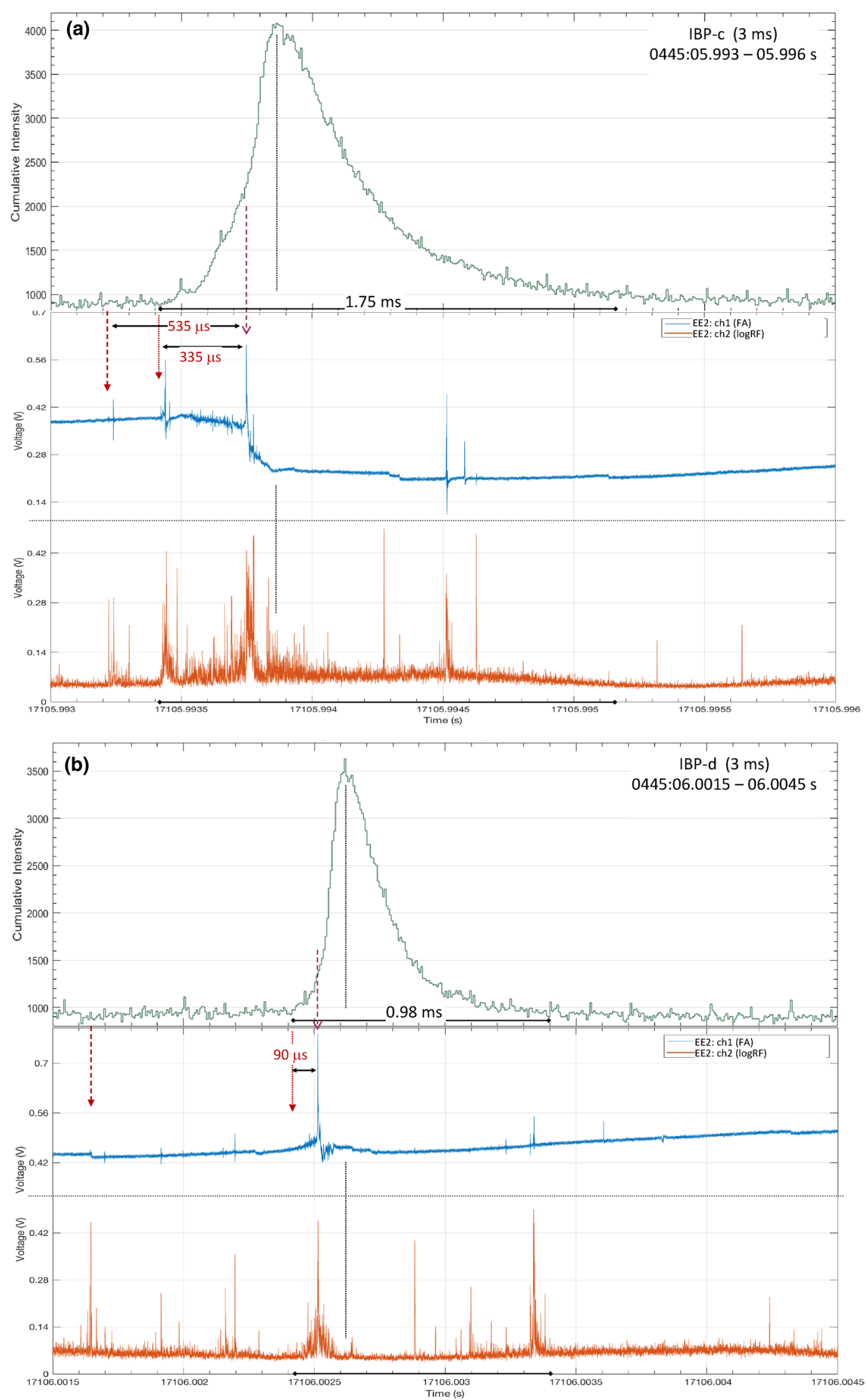
4 Fig. 10 a IB cluster IBP-c, as labeled in Fig. 7, expanded. b IB cluster IBP-d, as labeled in Fig. 7, expanded. The duration of enhanced luminosity for each IB cluster is indicated on the cumulative intensity plot and on the time axis (horizontal bar), and the dotted vertical bar indicates the time of peak intensity during each IB light burst. Purple arrows indicate time of each peak in E-change data. Red arrows indicate onset of large VHF emission within $1 \mathrm{~ms}$ before, and immediately before, the main IBP peak in each cluster

$1.55 \mathrm{~ms}$ and ending about the time that the enhanced VHF activity of each cluster ends.

Example 5 (Fig. 13) is much like Example 4, except that its IE is a weak event, not an NBE, although it is also observed with very weak detected light (cumulative intensity $<5 \%$ above background). The first three IB clusters show significant light detected for similar durations, averaging about $1.33 \mathrm{~ms}$, with similar timing relative to the E-change and VHF data as found in the IC flash examples presented herein.

\section{Discussion}

Kostinskiy et al. (2020) proposed a mechanism (called the KMS Mechanism hereinafter) to explain lightning initiation including the IE and the IB stage. Because it is new and relevant, we briefly examine whether the KMS Mechanism fits with the observations discussed above.

\subsection{KMS mechanism and IEs}

First we consider IEs and how they should be detected (or not) by our three main sensors: video camera (light intensity), LogRF sensor (VHF power), and E-change pulse(s) $\left(E_{100 \mathrm{~km}}\right)$. IEs in the KMS Mechanism are caused by a large number of almost simultaneous, positive streamer flashes, each with a length of $\sim 1 \mathrm{~m}$. (As background, we note that positive streamer flashes are composed of a large number of positive streamers, of the sort studied in laboratory spark experiments over many years and reviewed in Kostinskiy et al. (2020).) Strong IEs have many more positive streamer flashes than weak IEs. Note that the five example IC flashes studied were chosen partly because they have luminosity detected with IB clusters, thus we can expect that light emitted during their nearby IE should also be detectable in the same video data.

A single positive streamer flash emits weak visible light, as shown in Figs. 3 and 4 of Kostinskiy et al. (2015b). Thus, bright visible light is not expected for IEs, but weak visible light might be detected because there are many positive streamer flashes. The IEs in Examples 1, 3, 4 and 5 had weak light ( $\leq 5 \%$ above background) detected, while Example 2 had no light detected with its IE. Note that "no light detected" could be the result if weak light was emitted and obscured from the camera by dense clouds. Thus, the IE intensity findings are consistent with the KMS Mechanism.

Both weak and strong IEs should be detected with our VHF sensor because each positive streamer flash (with a length of $\sim 1 \mathrm{~m}$ ) radiates strongly in our VHF band (centered on wavelengths of $1.6 \mathrm{~m}$ ). Examples 1, 2 and 5 had weak IEs with VHF powers of $0.5,3$, and $1 \mathrm{~W}$. Examples 3 and 4 had strong IEs (both NBEs) with many positive streamer flashes

Table 1 Cumulative intensity, LogRF (VHF), and FA (E-change) parameters for Example 3 IC flash

\begin{tabular}{|c|c|c|c|c|c|c|c|}
\hline IB cluster & $\begin{array}{l}\text { Luminosity } \\
\text { duration (ms) }\end{array}$ & $\begin{array}{l}\text { Luminosity } \\
\text { time to peak } \\
(\mathrm{ms})\end{array}$ & $\begin{array}{l}\text { VHF onset to } \\
\text { luminosity onset } \\
(\mu \mathrm{s})\end{array}$ & $\begin{array}{l}\text { VHF onset to largest } \\
\text { E-change peak }(\mu \mathrm{s})\end{array}$ & $\begin{array}{l}\text { Number of FA pulses } \\
\text { total (before largest) }\end{array}$ & $\begin{array}{l}\text { VHF peak to } \\
\text { largest FA peak } \\
(\mu \mathrm{s})\end{array}$ & $\begin{array}{l}\text { FA pulse } \\
\text { during VHF } \\
\text { peak? }\end{array}$ \\
\hline IBP1 & 1.07 & 0.48 & 20 & 345 & $7(5)$ & 150 & Yes \\
\hline $\begin{array}{l}\text { IBP2 } \\
N B E\end{array}$ & 1.08 & 0.54 & 30 & 425 & $3(2)$ & 0 & Yes \\
\hline IBP3 & 1.36 & 0.47 & 70 & 290 & $3(1)$ & -60 & Yes \\
\hline IBP4 & 1.18 & 0.33 & 80 & 165 & $2(0)$ & 80 & No \\
\hline IBP5 & 0.85 & 0.40 & 80 & 180 & $1(0)$ & 0 & Yes \\
\hline $\begin{array}{l}\text { IBP-a } \\
\text { LINET }\end{array}$ & 1.72 & 0.52 & 0 & 260 & $2(1)$ & -5 & Yes \\
\hline IBP-bb & 0.95 & 0.60 & 120 & 110 & $1(0)$ & 0 & Yes \\
\hline IBP-b & 1.65 & 0.70 & 0 & 520 & $2(1)$ & 0 & Yes \\
\hline IBP-c & 1.75 & 0.42 & 0 & 335 & $4(2)$ & -10 & Yes \\
\hline IBP-d & 0.98 & 0.20 & 0 & 90 & $1(0)$ & 0 & Yes \\
\hline Avg. & 1.26 & 0.47 & 40 & 272 & & 15.5 & \\
\hline
\end{tabular}




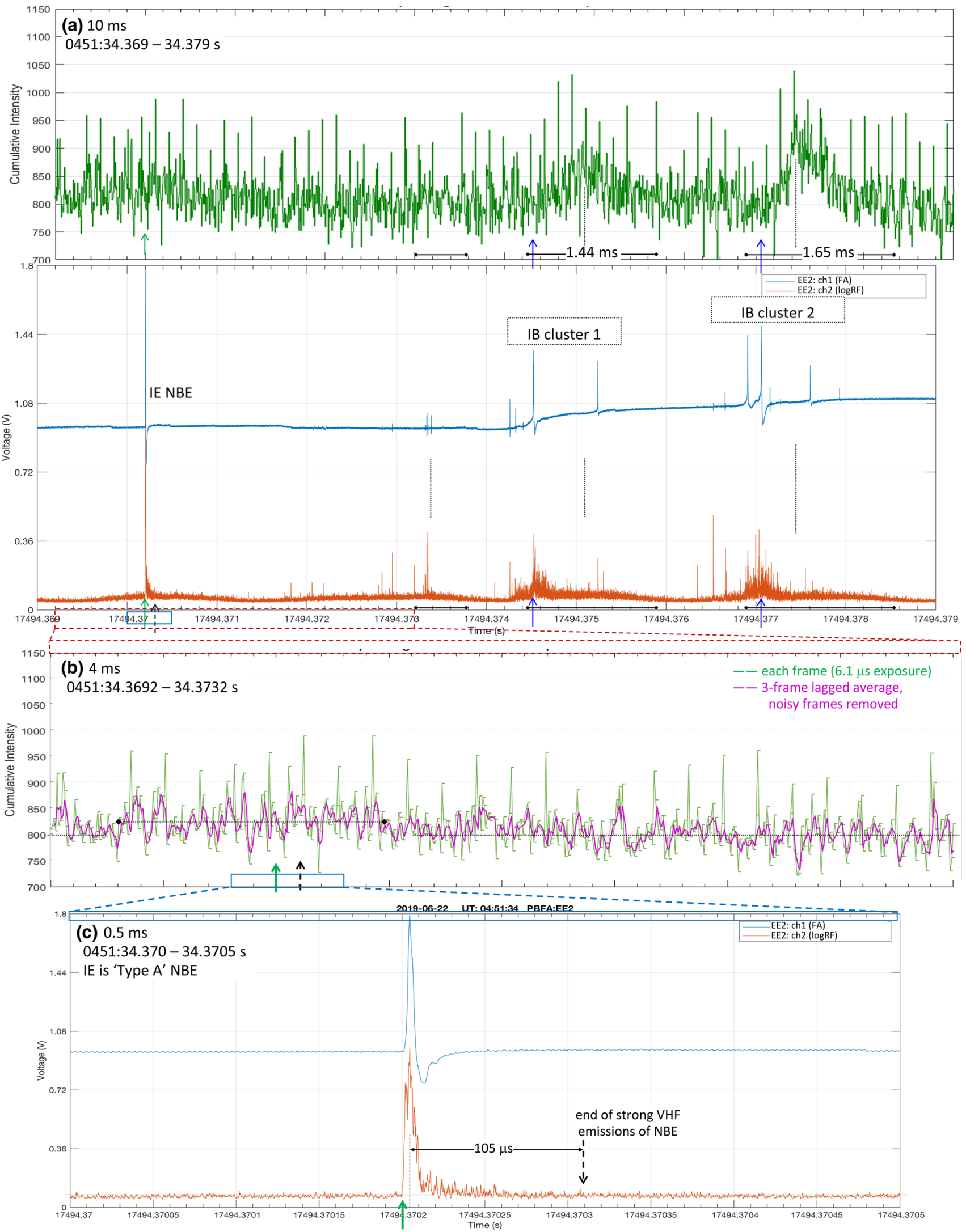


4Fig. 11 Beginning of Example 4, IC flash at 0451:34 UT on 22 June $2019,18.1 \mathrm{~km}$ distance (19.2 km slant range) from camera. a First $10 \mathrm{~ms}$ of E-change (FA) and VHF (logRF) data, with cumulative intensity at top. Green arrow indicates beginning of the initiating event (IE), a positive NBE, and black arrow $110 \mu$ s later indicates the end of strong VHF emissions associated with the IE. Blue arrows mark times of the largest classic IBP in the earliest two large IB clusters. Durations of IB intensity enhancements are given, and vertical lines are times of peak intensity. Red and blue rectangles indicate times in lower figure parts. b Expanded view of first $4 \mathrm{~ms}$ of cumulative intensity; frame area at $18.1 \mathrm{~km}$ distance is $9.3 \mathrm{~km}$ across by $12.0 \mathrm{~km}$ above ground. Intensities average $3 \%$ above background for $\sim 1200 \mu$ s (dashed horizontal line) before, during, and after the IE. c FA and $\operatorname{logRF}$ data for the first $500 \mu \mathrm{s}$, showing the IE was a Type A Narrow Bipolar Event. Event altitude is $7.85 \mathrm{~km}$, range-normalized peak E-change amplitude is $3.7 \mathrm{~V} / \mathrm{m}$, coincident with the peak VHF emission which has estimated source power of $990 \mathrm{~W}$. Dotted horizontal through $\log \mathrm{RF}$ data indicates approximate (upper) background level

and VHF powers of $43 \mathrm{~W}$ and $990 \mathrm{~W}$. Thus the IE VHF power findings are consistent with the KMS Mechanism.

An individual positive streamer flash is not likely to be observable in the E-change data, since our E-change sensors detect charge motions with lengths $>120 \mathrm{~m}$. However, Kostinskiy et al. (2020) hypothesized that a strong IE (such as an NBE) will have many nearly simultaneous positive streamer flashes distributed through a volume with a characteristic scale of $500 \mathrm{~m}$. Hence, the superposition of the positive streamer currents can appear to have a length $\geq 100 \mathrm{~m}$, thereby making a strong IE detectable by an E-change sensor, while a weak IE would not be detected. The IEs in Examples 1 and 5 had no accompanying E-change pulses; the IEs in Examples 2 and 3 (a weak NBE) both had E-change pulses with $E_{100 \mathrm{~km}}$ amplitudes of $0.3 \mathrm{~V} / \mathrm{m}$ and Example 4 (a fairly strong NBE) had an accompanying E-change pulse with $E_{100 \mathrm{~km}}$ of $3.7 \mathrm{~V} / \mathrm{m}$. These data seem consistent with the KMS Mechanism.

\subsection{KMS mechanism and visible light intensity of IB clusters}

The KMS Mechanism postulates that IB pulses develop from the positive streamer flashes (produced by the IE) in three distinct stages.

First, many of the positive streamer flashes transition to UPFs (Unusual Plasma Formations), which are hot conducting channels with lengths of $1-30 \mathrm{~cm}$. Because of their short lengths, it is doubtful that these transitions are seen by our sensors. Note that UPFs were first observed by Kostinskiy et al. (2015a), who found that UPFs "can occur in the absence of spark leaders and appear to be manifestations of collective processes building, essentially from scratch, a complex hierarchical network of interacting channels at different stages of development (some of which are hot and live for milliseconds)." Although the processes causing the development of UPFs were not determined, the existence of UPFs was demonstrated in multiple experiments.

Second, UPFs merge into bigger and bigger three-dimensional (3-D) UPF networks. It is important to realize that many positive streamer flashes are joined into each 3-D UPF network. As these networks get to meter scales, our VHF sensors will begin to detect the mergers. We expect that when two UPF networks merge, one with a length scale of $\sim 100 \mathrm{~m}$ and one with a much shorter length, the merger will be seen with the E-change sensor as a narrow IBP; these are called "preparatory mergers" in the KMS Mechanism. Thus we expect to see many small VHF pulses as the flash initiation develops and to occasionally see narrow IBPs when larger UPF networks are involved. The merging of many hundreds or thousands of UPFs into UPF networks and of small UPF networks into larger UPF networks provides the copious VHF pulses detected before the first classic IBP. Concerning visible light production, because the UPFs are hot channels, they provide the increasing visible light seen in the 200-500 $\mu$ s just prior to the first classic IBP.

Third, when two UPF networks with length scales of $100 \mathrm{~m}$ merge, the first classic IBP occurs and will be easily seen with the E-change sensor, both because of its length and because of the kA currents that are known to flow in classic IBPs. On the visual side, the kA current of the classic IBP will make a strong burst of light. In the KMS mechanism, each subsequent classic IBP is caused by another large UPF network joining the networks that merged to make the earlier classic IBPs. Thus the fact that there is visible light detected with each classic IB pulse is consistent with the KMS mechanism.

Finally, it is important to consider the strong VHF emissions that occur for a few hundred microseconds after each classic IBP; Marshall et al. (2019) noted these VHF emissions, marked them with question marks in their Fig. 6, and wondered about their source. Based on the KMS Mechanism, we suggest that the large current surge of the classic IBP will emit substantial VHF pulses after the E-change peak of a classic IBP as the IBP current passes through the many twists and turns of the merging 3-D UPF networks. The KMS Mechanism estimated the lengths of conducting channels in 3-D UPF networks and found $300-3000 \mathrm{~m}$ for weaker classic IBPs and 1700-17,000 $\mathrm{m}$ for larger amplitude classic IBPs (Kostinskiy et al. 2020). As a rough estimate of the duration of the VHF pulses emitted just after the IBP E-change peak, let the total length of the merging UPF networks be $3000 \mathrm{~m}$ and assume a discharge speed of $10^{7} \mathrm{~m} / \mathrm{s}$, then the duration would be $300 \mu$ s. This time is in reasonable agreement with the duration of VHF emissions observed after classic IBPs (Figs. 7, 8, 9, 10). In particular, in Example 3, the KMS Mechanism would attribute the strong VHF 

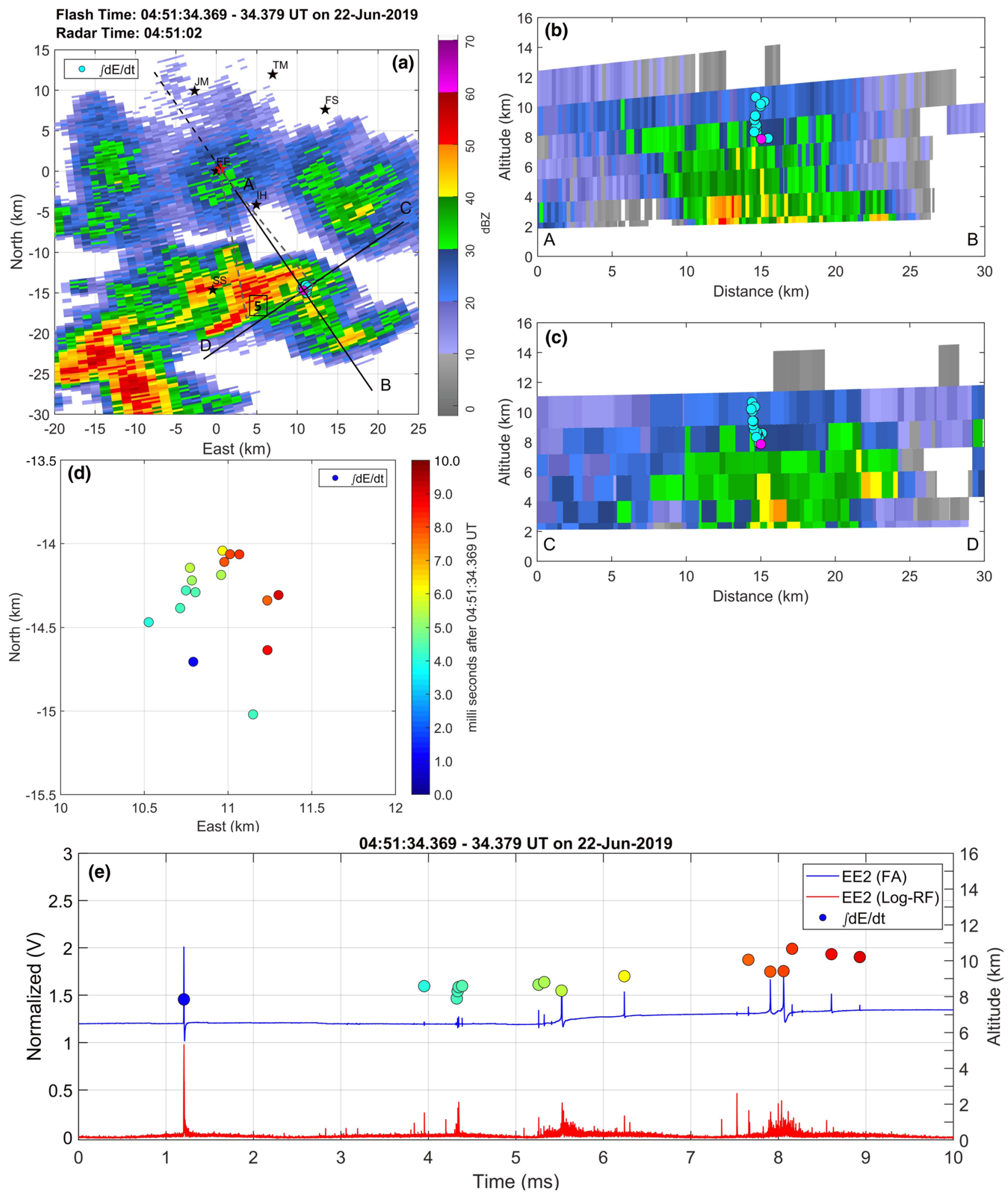

Fig. 12 Radar reflectivity and pulse locations for Example 4, IC flash at 0451:34 UT on 22 June 2019. Radar data are $\sim 9 \mathrm{~min}$ and $\sim 4.5 \mathrm{~min}$ after those in Figs. 4 and 6; storm motion is $\sim 35 \mathrm{~km} / \mathrm{h}$ eastward. a Plan view of low-level PPI scan, with locations of pulses for the first $10 \mathrm{~ms}$ of the flash overlaid. (First pulse, the NBE, is magenta, others are cyan dots.) Black stars are sensor sites, red star is camera location, and black lines indicate cross-section locations. Box enclosing
[5] is location of early pulses of flash Example 5. b Radar cross-section along the line of sight of the camera toward the start of IC flash. c Radar cross-section perpendicular to $\mathbf{b}$ and approximately along video camera image. d Plan view of first $10 \mathrm{~ms}$ of pulse locations, color-coded by time, for expanded area of initiation. e Time series of normalized E-change (FA), VHF ( $\operatorname{logRF}$ ), and pulse altitudes (colorcoded as in d) for the first $10 \mathrm{~ms}$ of flash 
pulses-starting just after the E-change peak of each classic IBP and ending with the vertical dot/dash line that marks the peak in visible luminosity associated with each IB clusterto the current traveling through the merged UPF networks.

\section{Conclusions}

In this study, we examine high-speed video data, with $6.1 \mu$ s exposure per frame and $6.66 \mu$ s frame interval, from the initiating event (IE) through several of the following initial breakdown (IB) pulses of five typical IC lightning flashes at 16-19 km range. The flashes chosen are good cases in which to investigate IE luminosity because there is luminosity detected with the subsequent nearby IB clusters. Analyses are based on cumulative light intensity data (350-1060 $\mathrm{nm}$ wavelength range) across entire video frames, along with time-correlated E-change (fast antenna) and VHF (log RF) data for the first 10-50 ms. Pulse locations and relevant radar reflectivity data for the storms provide context information.

Four of the five IC flashes studied have very slight amount of light ( $\leq 5 \%$ above background cumulative intensity) detected at the time of the IE; the weak IE of Example 2 had no detected luminosity increase. One of the luminous IEs was a 'hump-type' positive NBE (Example 3) and one was a strong Type A NBE (Example 4); the other two weakly luminous IEs were weak events in E-change and VHF data. Based on these observations of only a little or no visible luminosity coincident with IEs, we conclude that the IEs of all these flashes did not have a bright, hot, conducting channel. This finding is in agreement with Kostinskiy et al. (2020) in which the IE is a rapid series of positive streamer flashes. In these IC flashes, the timing of the onset, peak, and fall-off of weak luminosity relative to the electromagnetic data are qualitatively similar in the four IEs with detected light. Overall durations of the IE luminosity enhancements, although very weak, are 180-600 $\mu$ s with an average of $450 \mu \mathrm{s}$. Based on these analyses, we conclude that IEs of IC flashes are probably always slightly luminous processes, producing light that is sometimes detectable in the 350-1060 $\mu \mathrm{m}$ wavelength range even with short exposure times and even when viewed through cloud. The very weak luminosity we observed during the IE of IC flashes may also help better understand 'fast positive breakdown' of the type that has been observed with broadband interferometers (e.g. Rison et al. 2016). However, to us, such a connection faces a basic difficulty for our data: fast positive breakdown implies that the IE is an NBE (that is, the NBE is caused by fast positive breakdown), but only two of our five IC flashes initiated with an NBE. The recent work by Scholten et al. (2021) using other detailed lightning mapping systems, along with more recent studies using broadband interferometry, may be better poised to place these results within the context of fast positive breakdown.

Among the five IC flash examples, light was detected coincident with as many as five positive NBEs or possible NBEs:

1. a Type 11 CID that occurred after the weak IE (in Example 1) had a weak, but clear, light intensity increase of $\sim 9 \%$; this event may instead have been a classic IBP of an IB cluster.

2. a Type D NBE that occurred $1.23 \mathrm{~ms}$ after a weak IE (in Example 2) had a light intensity increase lasting for about $0.81 \mathrm{~ms}$ that peaked about $30 \%$ above background and appears similar to IB clusters occurring after it; this event may instead have been a classic IBP of an IB cluster.

3. a positive 'hump-type' NBE that is the IE (Example 3), with peak intensity about $5-7 \%$ above background and intensity above background for $300 \mu \mathrm{s}$.

4. a Type A NBE (IBP2, Example 3) that is $2.35 \mathrm{~ms}$ after the IE and appears much like other IB clusters that surround it in time, with intensity increase for about $1.08 \mathrm{~ms}$ duration and peak about $45 \%$ above background value; this event may instead have been a classic IBP of an IB cluster.

5. a positive Type A NBE that is the IE (Example 4), with peak intensity $<5 \%$ above background.

To our knowledge, these are the first published data showing visible light detected with any positive NBEs, including two that initiate IC flashes.

As has been found in earlier studies (e.g., Wilkes et al. 2016; Stolzenburg et al. 2016), significant light is also detected with IC-type IB pulses in the flashes studied here. Data for 18 IB clusters (with the E-change data of each cluster lasting $<750 \mu$ s and typically consisting of several narrow IB pulses leading up to one or two classic IB pulses), show clear increases in luminosity. The intensity increase begins near or just after the E-change and VHF data of each IB cluster begin to vary, with the largest pulses in the E-change and VHF data occurring during the intensity increase. This latter sequence is in agreement with the findings of Stolzenburg et al. (2016) for individual IC-type IB pulses in E-change and luminosity data. The typical IB cluster shows increasing cumulative intensity for $400-800 \mu$ s, with peak close to the time when largest VHF emissions cease, then a slower intensity fall-off for 500-1000 $\mu$ s; during the time of decreasing intensity there is usually also appreciable, but weaker, VHF activity. After the light of the IB cluster decays to background, usually no light is detected until the next IB cluster begins. However, some short duration, fast, and relatively small amplitude pulses in the VHF and E-change data during these same IB stages do have corresponding increases in luminosity detected by 
(a) $10 \mathrm{~ms}, 0452: 27.370-27.380 \mathrm{~s}$

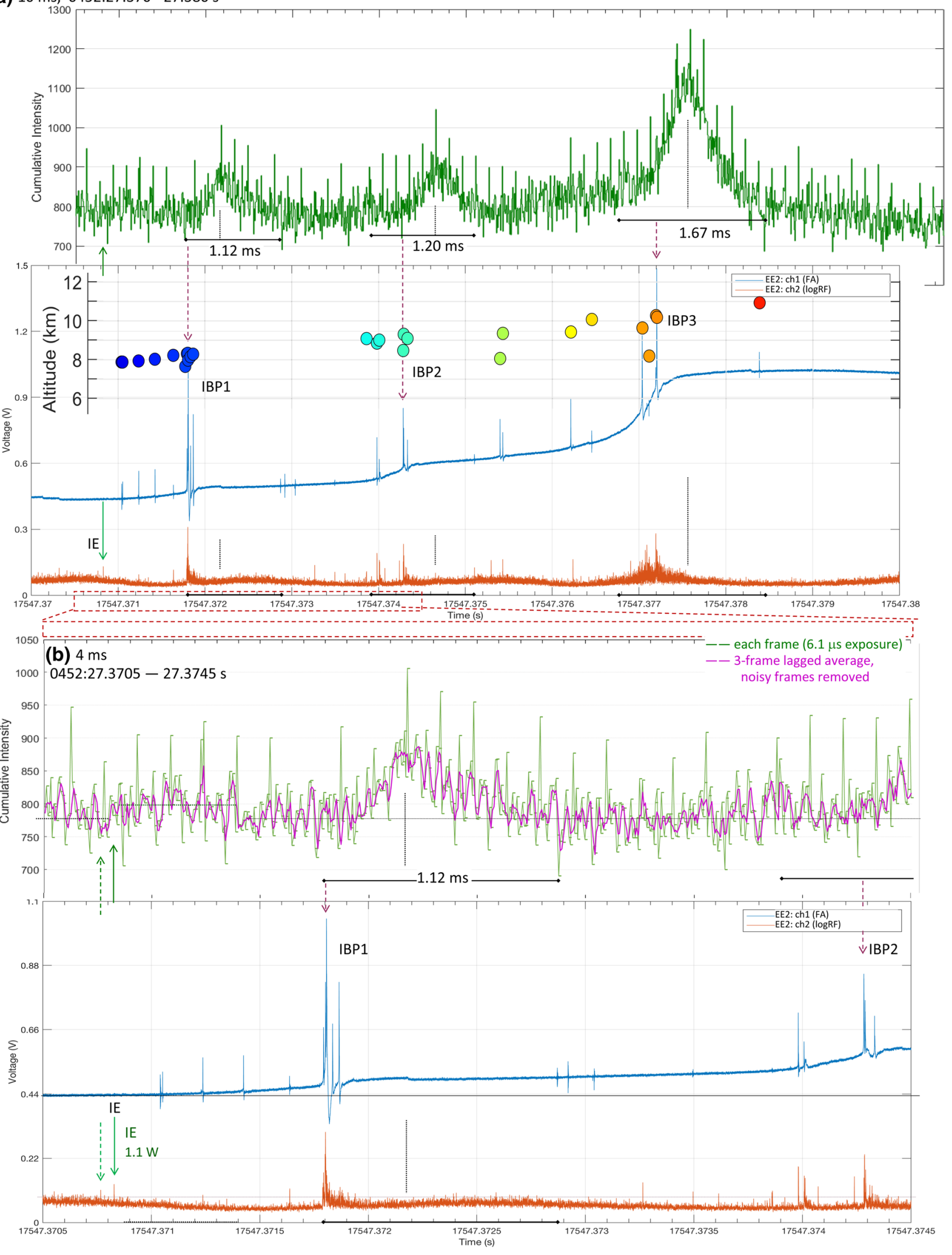


4Fig. 13 Beginning of Example 5, IC flash at 0452:27 UT on 22 June 2019, $18.1 \mathrm{~km}$ from camera, in same storm as shown in Figs. 4, 6, and 12. a First $10 \mathrm{~ms}$ of E-change (FA) and VHF (logRF) data, with cumulative intensity data at top. Green arrow indicates the initiating event (IE), not detected in E-change data nor located with $\mathrm{dE} / \mathrm{dt}$ data. Purple arrows mark the times of the largest classic IBP in the earliest three IB clusters. Durations of IBP intensity enhancements are given, and vertical lines are times of peak intensity. In the overlay, circles are pulse altitudes, color-coded by time, with an altitude scale inside left axis. b Expanded view of first $4 \mathrm{~ms}$ (red rectangle in a). Intensity values average $~ 3 \%$ above background (dotted horizontal line) for $\sim 500 \mu$ s after the IE; peak intensity is $7 \%$ above background. With the first IB cluster (IBP1), intensity enhancement begins near the time of FA peak of classic IBP and rises during the next $500 \mu$ s of largest VHF emissions; peak intensity is $13 \%$ above background. IBP1 location is $<500 \mathrm{~m}$ horizontal distance from the IE and $\sim 600 \mathrm{~m}$ higher, at altitude $8.3 \mathrm{~km}$. Dashed green arrows indicate a relatively weak VHF event detected $90 \mu$ s before the IE but only at this sensor; it is interpreted as local noise but may have been the flash IE

the camera. Durations of the cumulative intensity increase with IB clusters in these IC flashes are $0.52-1.75 \mathrm{~ms}$ and average $1.21 \mathrm{~ms}$.

Two other features of this dataset are worth noting. Firstly, we compared the data from the five IC flashes to the KMS mechanism for lightning initiation (Kostinskiy et al. 2020). The KMS mechanism predictions for visible light, E-change pulses, and VHF pulses during the IE and IB clusters were in reasonable agreement with the IC flash data presented herein. Secondly, four of the IC flashes analyzed herein occurred in the same storm system within 9 min and $15 \mathrm{~km}$ of each other. This night-time storm had developed with weak forcing along a long-lived and weakening outflow boundary, and maximum radar echo tops were only about $14 \mathrm{~km}$. These four IC flashes exhibited similar sequences of rapid upward development, ascending by $3.0-3.5 \mathrm{~km}$ in altitude, early in the IB stage, after the first few IB clusters and within $8 \mathrm{~ms}$ after the IE occurred near $8 \mathrm{~km}$ altitude. This sequence of rapid upward development may be common for typical IC flashes, even in storms with weakly organized, relatively shallow, midlatitude convection (as studied herein).

This study is the first comparison of lightning initiation that includes VHF, E-change, and high-speed video data for natural flashes. To us, the most intriguing result of these comparisons is that the character of VHF activity appears closely tied to luminosity during IC IB stages. This association occurs to such an extent that we hypothesize that any events which yield VHF emissions for tens of microseconds or longer are likely also emitting detectable light. Tests of this hypothesis certainly require further study.

Acknowledgements The authors extend a special thank you to Richard Orville for his help and guidance with all aspects of designing and proposing this study, and to the staff at TAMU who were actively supportive of the proposal process. We appreciate all the site hosts: Jan Murray, Cherry and Scott Watkins, Crystal and William McKenzie,
Martha Mills and North Delta School, MRRPMDC Orchards, UM Biological Field Station, and UM EE Department. We also thank the Inn at Ole Miss staff for hosting us in the top corner suite. We are eternally grateful to Sumedhe Karunarathne for earlier instrument fabrication, programming, and continual support of our research. LINET data were generously provided by Dirk Brändlein, nowcast GmbH. Very special appreciation is extended to Nicholas Anderson at NSF-PDM for going way way beyond the call of duty in helping us to manage the many complications and tricky circumstances faced surrounding this project; it truly would not have been possible without his attention and action. This work is a part of NSF Grant AGS-1745931. All UMacquired data used herein are freely available from the corresponding author. Radar data are available from the NOAA National Climatic Data Center online repository.

Author contributions All authors contributed to the research data collection. Material preparation and data analyses were performed by MS, TCM, and SB. The first draft of the manuscript was written by MS, with major components added by TCM. All authors commented on versions of the manuscript, and all authors read and approved the submitted and revised manuscript versions. MS was principal and TCM co-principal in the study design, funding acquisition, and resource maintenance for the project.

Funding This study was funded by the US National Science Foundation, Grant AGS-1745931.

Data availability All data used herein are available from the corresponding author.

\section{Declarations}

Conflicts of interest The authors have no conflicts of interest to declare that are relevant to the content of this article.

Open Access This article is licensed under a Creative Commons Attribution 4.0 International License, which permits use, sharing, adaptation, distribution and reproduction in any medium or format, as long as you give appropriate credit to the original author(s) and the source, provide a link to the Creative Commons licence, and indicate if changes were made. The images or other third party material in this article are included in the article's Creative Commons licence, unless indicated otherwise in a credit line to the material. If material is not included in the article's Creative Commons licence and your intended use is not permitted by statutory regulation or exceeds the permitted use, you will need to obtain permission directly from the copyright holder. To view a copy of this licence, visit http://creativecommons.org/licenses/by/4.0/.

\section{References}

Bandara S, Marshall TC, Karunarathne S, Karunarathne N, Siedlecki R, Stolzenburg M (2019) Characterizing three types of negative narrow bipolar events in thunderstorms. Atmos Res 227:263-279. https://doi.org/10.1016/j.atmosres.2019.05.013

Bandara S, Marshall TC, Karunarathne S, Stolzenburg M (2020) Electric field change and VHF waveforms of positive Narrow Bipolar Events in Mississippi thunderstorms. Atmos Res 243:105000. https://doi.org/10.1016/j.atmosres.2020.105000

Betz H-D, Schmidt K, Oettinger P, Wirz M (2004) Lightning detection with 3-D discrimination of intracloud and cloud-to-ground discharges. Geophys Res Lett 31:L11108. https://doi.org/10.1029/ 2004GL019821 
Betz H-D, Marshall TC, Stolzenburg M, Schmidt K, Oettinger WP, Defer E, Konarski J, Laroche P, Dombai F (2008) Detection of in-cloud lightning with VLF/LF and VHF networks for studies of the initial discharge phase. Geophys Res Lett 35:L23802. https:// doi.org/10.1029/2008GL035820

Campos LZS, Saba MMF (2013) Visible channel development during the initial breakdown of a natural negative cloud-to-ground flash. Geophys Res Lett 40:4756-4761. https://doi.org/10.1002/ grl.50904

Chapman R, Marshall T, Karunarathne S, Stolzenburg M (2017) Initial electric field changes of lightning flashes in two thunderstorms. J Geophys Res Atmos 122:3718-3732. https://doi.org/10.1002/ 2016JD025859

Coleman LM, Marshall TC, Stolzenburg M, Hamlin T, Krehbiel PR, Rison W, Thomas RJ (2003) Effects of charge and electrostatic potential on lightning propagation. J Geophys Res Atmos 108(D9):4298. https://doi.org/10.1029/2002JD002718

Jerauld J, Uman MA, Rakov VA, Rambo KJ, Jordan DM, Schnetzer GH (2008) Electric and magnetic fields and field derivatives from lightning stepped leaders and first return strokes measured at distances from 100 to 1000 m. J Geophys Res Atmos 113:D17111. https://doi.org/10.1029/2008JD010171

Karunarathne S, Marshall TC, Stolzenburg M, Karunarathna N, Vickers LE, Warner TA, Orville RE (2013) Locating initial breakdown pulses using electric field change network. J Geophys Res Atmos 118:7129-7141. https://doi.org/10.1002/jgrd.50441

Karunarathne S, Marshall TC, Stolzenburg M, Karunarathna N (2015) Observations of positive narrow bipolar pulses. J Geophys Res Atmos 120:7128-7143. https://doi.org/10.1002/2015JD023150

Kitagawa N, Brook M (1960) A comparison of intracloud and cloud-toground lightning discharges. J Geophys Res Atmos 65(4):11891201. https://doi.org/10.1029/JZ065i004p01189

Kostinskiy AY, Syssoev VS, Bogatov NA, Mareev EA, Andreev MG et al (2015a) Observation of a new class of electric discharges within artificial clouds of charged water droplets and its implication for lightning initiation within thunderclouds. Geophys Res Lett 42:8165-8171. https://doi.org/10.1002/2015GL065620

Kostinskiy AY, Syssoev VS, Mareev EA, Rakov VA, Andreev MG, Bogatov NA et al (2015b) Electric discharges produced by clouds of charged water droplets in the presence of moving conducting object. J Atmos Solar-Terrestrial Phys 135:36-41. https://doi.org/ 10.1016/j.jastp.2015.10.006

Kostinskiy AY, Marshall TC, Stolzenburg M (2020) The mechanism of the origin and development of lightning from initiating event to initial breakdown pulses (v2). J Geophys Res Atmos 125:e2020JD033191. https://doi.org/10.1029/2020JD033191

Leal AFR, Rakov VA, Rocha BRP (2019) Compact intracloud discharges: new classification of field waveforms and identification by lightning locating systems. Electr Power Syst Res 173:251262. https://doi.org/10.1016/j.epsr.2019.04.016

Le Vine DM (1980) Sources of strongest RF radiation from lightning. J Geophys Res 85:4091-4095. https://doi.org/10.1029/JC085iC07p 04091

Lyu F, Cummer SA, Qin Z, Chen M (2019) Lightning initiation processes imaged with very high frequency broadband interferometry. J Geophys Res Atmos 124:2994-3004. https://doi.org/10.1029/ 2018JD029817

Marshall TC, Stolzenburg M, Karunarathne S, Cummer S, Lu G, Betz H-D, Briggs M (2013) Initial breakdown pulses in intracloud lightning flashes and their relation to terrestrial gamma ray flashes. J Geophys Res Atmos 118:10907-10925. https://doi.org/ 10.1002 jgrd. 50866

Marshall T, Stolzenburg M, Karunarathna N, Karunarathne S (2014) Electromagnetic activity before initial breakdown pulses of lightning. J Geophys Res Atmos 119:12558-12574. https://doi.org/10. 1002/2014JD022155
Marshall TC, Bandara S, Karunarathne N, Karunarathne S, Kolmašová I, Siedlecki R, Stolzenburg M (2019) A study of lightning flash initiation prior to the first initial breakdown pulse. Atmos Res 217:10-23. https://doi.org/10.1016/j.atmosres.2018.10.013

Nag A, DeCarlo BA, Rakov VA (2009) Analysis of microsecond- and submicrosecond-scale electric field pulses produced by cloud and ground lightning discharges. Atmos Res 91(2-4):316-325. https:// doi.org/10.1016/j.atmosres.2008.01.014

Nag A, Rakov VA, Tsalikis D, Cramer JA (2010) On phenomenology of compact intracloud lightning discharges. J Geophys Res 115:D14115. https://doi.org/10.1029/2009JD012957

Rison W, Thomas RJ, Krehbiel PR, Hamlin T, Harlin J (1999) A GPSbased three dimensional lightning mapping system: initial observations in central New Mexico. Geophys Res Lett 26:3573-3576. https://doi.org/10.1029/1999GL010856

Rison W, Krehbiel PR, Stock MG, Edens HE, Shao XM, Thomas RJ et al (2016) Observations of narrow bipolar events reveal how lightning is initiated in thunderstorms. Nat Commun 7(1):10721. https://doi.org/10.1038/ncomms 10721

Scholten O, Hare BM, Dwyer J, Sterpka C, Kolmašová I, Santolík O et al (2021) The initial stage of cloud lightning imaged in highresolution. J Geophys Res Atmos 126:e2020JD033126. https:// doi.org/10.1029/2020JD033126

Shi D, Wang D, Wu T, Takagi N (2019) Temporal and spatial characteristics of preliminary breakdown pulses in intracloud lightning flashes. J Geophys Res Atmos 124:12901-12914. https://doi.org/ 10.1029/2019JD031130

Smith EM, Marshall TC, Karunarathne S, Siedlecki R, Stolzenburg M (2018) Initial breakdown pulse parameters in intracloud and cloud-to-ground lightning flashes. J Geophys Res Atmos 123:2129-2140. https://doi.org/10.1002/2017JD027729

Stolzenburg M, Marshall TC, Karunarathne S, Karunarathna N, Vickers LE, Warner TA et al (2013) Luminosity of initial breakdown in lightning. J Geophys Res Atmos 118:2918-2937. https://doi. org/10.1002/jgrd.50276

Stolzenburg M, Marshall TC, Karunarathne S, Karunarathna N, Orville RE (2014) Leader observations during the initial breakdown stage of a lightning flash. J Geophys Res 119:12198-12221. https://doi. org/10.1002/2014JD021994

Stolzenburg M, Marshall TC, Karunarathne S, Orville RE (2016) Luminosity with intracloud-type lightning initial breakdown pulses and terrestrial gamma-ray flash candidates. J Geophys Res Atmos 121:10919-10936. https://doi.org/10.1002/2016JD025202

Stolzenburg M, Marshall TC, Karunarathne S (2020) On the transition from initial leader to stepped leader in negative cloud-to-ground lightning. J Geophys Res Atmos 125:e2019JD031765. https://doi. org/10.1029/2019JD031765

Weidman CD, Krider EP (1979) The radiation field waveforms produced by intracloud lightning discharge processes. J Geophys Res 84(C6):3159-3164. https://doi.org/10.1029/JC084iC06p03159

Wilkes RA, Uman MA, Pilkey JT, Jordan DM (2016) Luminosity in the initial breakdown stage of cloud-to-ground and intracloud lightning. J Geophys Res Atmos 121:1236-1247. https://doi.org/ 10.1002/2015JD024137

Willett JC, Bailey JC, Krider EP (1989) A class of unusual lightning electric field waveforms with very strong high-frequency radiation. J Geophys Res 94:16255. https://doi.org/10.1029/JD094iD13p16255

Wu T, Yoshida S, Ushio T, Kawasaki Z, Wang D (2014) Lightninginitiator type of narrow bipolar events and their subsequent pulse trains. J Geophys Res 119:7425-7438. https://doi.org/10.1002/ 2014JD021842

Publisher's Note Springer Nature remains neutral with regard to jurisdictional claims in published maps and institutional affiliations. 